\title{
Detecting ecological regime shifts from transect data
}

\author{
Delphi F. L. Ward iD ${ }^{1,2,5}$ Simon Wotherspoon, ${ }^{1,3}$ Jessica Melbourne-Thomas (iD, 2,3 \\ Jessica HaApkylä, ${ }^{4}$ and Craig R. Johnson \\ ${ }^{1}$ Institute for Marine and Antarctic Studies, University of Tasmania, Private Bag 129, Hobart, Tasmania 7001 Australia \\ ${ }^{2}$ Antarctic Climate \& Ecosystems Cooperative Research Centre, University of Tasmania, Private Bag 80, Hobart, Tasmania 7001 Australia \\ ${ }^{3}$ Australian Antarctic Division, Department of the Environment and Energy, 203 Channel Highway, Kingston, Tasmania 7050 Australia \\ ${ }^{4}$ School of Marine and Tropical Biology, ARC Centre of Excellence for Coral Reef Studies, James Cook University, Townsville, \\ Queensland 4811 Australia
}

\begin{abstract}
Timely detection of ecological regime shifts is a key problem for ecosystem managers, because changed ecosystem dynamics and function will usually necessitate a change in management strategies. However, currently available methods for detecting regime shifts depend on having multiple long time series data from both before and after the regime shift. This data requirement is prohibitive for many ecosystems. Here, we present a new approach for detecting regime shifts from one-dimensional spatial (transect) data from just a single time step either side of the transition. Characteristic length scale (CLS) estimation is a method of attractor reconstruction combined with nonlinear prediction that enables identification of the emergent scale at which deterministic behavior of the system is best observed. Importantly, previous studies show that a fundamental change in ecosystem dynamics, from one domain of attraction to another, is reflected in a change in the CLS, i.e., the approach enables distinguishing regime shifts from variability in dynamics around a single attractor. Until now the method required highly resolved two-dimensional spatial data, but here we adapted the approach so that the CLS can be estimated from one-dimensional transect data. We demonstrate its successful application to both model and real ecosystem data. In our model test cases, we detected change in the CLS in cases where the shape (topology) of the interaction network had changed, leading to a shift in community composition. In an examination of benthic transect data from four Indonesian coral reefs, changes in the CLS for two of the reefs indicate a regime shift. This new development in estimating CLSs makes it possible to detect regime shifts in systems where data are limited, removing ambiguity in the interpretation of community change.
\end{abstract}

Key words: attractor reconstruction; characteristic length scale; coral disease; coral reefs; critical transitions; ecosystem change; emergent spatial patterns; nonlinear prediction; phase shifts; spatial data.

\section{INTRODUCTION}

Increased anthropogenic pressure is driving change in most ecosystems (Walther et al. 2002, Walther 2010, Hughes et al. 2017, Pecl et al. 2017). Yet as the drivers and nature of ecosystem change are varied and interdependent, characterizing and quantifying the degree and type of change remains a challenge. Ecosystem change can take many forms. Losses and invasions of species with particular qualities can completely disrupt the dynamics of some ecosystems, but have little observable impact in others (Ricciardi et al. 2013). Changes in the frequency or type of disturbance events can force changes in any number of directions and can interact with other ecological changes in complex ways (Sousa 1984, Pascual and Guichard 2005, Turner 2010). While some ecosystem changes may be straightforward to detect (for example, the addition or loss of species), whether an observed change constitutes a fundamental change in system structure and dynamics, a transition from one regime to another, is much more difficult to determine (deYoung et al. 2008, Litzow et al. 2016).

The distinction between ecosystem state and regime is important. The state of an ecosystem is essentially its status at a point in time, for example, the population sizes (or other

Manuscript received 26 October 2017; revised 5 March 2018; accepted 29 March 2018. Corresponding Editor: Lewi Stone.

${ }^{5}$ E-mail: delphi.ward@utas.edu.au state variable) of component species (Biggs et al. 2012). Ecosystem state changes dynamically and continuously; for example, seasonal oscillations between high and low biomass states, or multiannual cycles in population sizes. However, a change in regime constitutes a fundamental change in the dynamics of the ecosystem, such that the system functions in a different way than previously (Scheffer and Carpenter 2003, Biggs et al. 2012). Importantly, there may be many possible ecosystem states within the same regime. For example, Fig. 1 shows a well-studied example of a system that has large changes in state within a single regime. This type of intrinsic system behavior complicates the detection of regime shifts based on changes in state. Consequently, changes in ecosystem state alone are not evidence of a change in regime. A shift from one regime to another is difficult to demonstrate because it requires evidence that fundamental dynamics, e.g., the nature of species interactions, or more formally, the underlying attractor for the system (see Glossary in Box 1), have changed. Harnessing information about system dynamics inferred from emergent spatial pattern is likely to be one practical means of overcoming this challenge (Marcos-Nikolaus et al. 2002, Hammond and Kolasa 2014).

Regime shifts can have significant consequences for ecosystem function and service delivery (Nystrom et al. 2012, Graham et al. 2013, Travis et al. 2014, Ainsworth and Mumby 2015, Selkoe et al. 2015). Thus, identification of regime shifts is vital for effective ecosystem management, managing an 

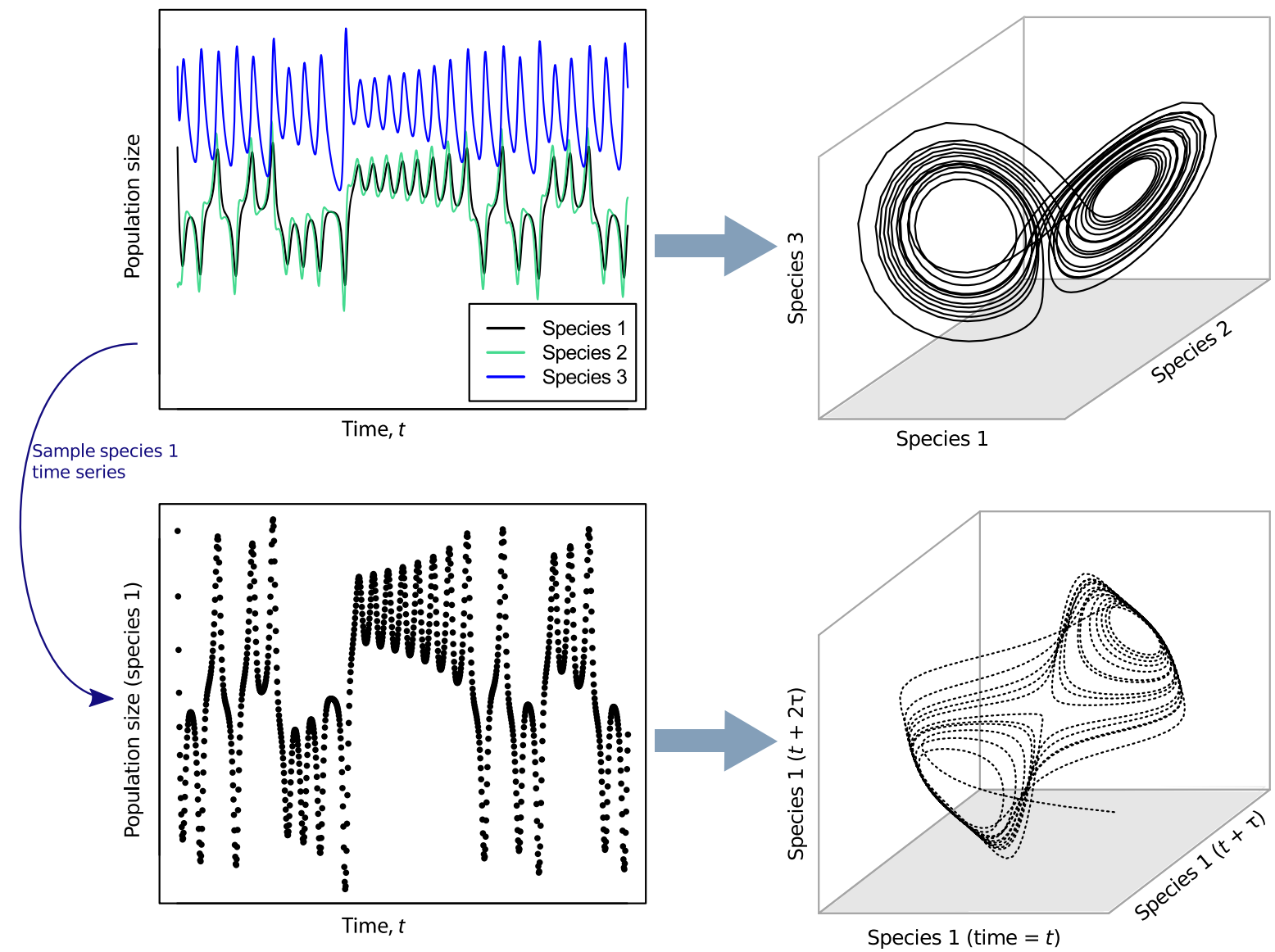

FIG. 1. Schematic representation of attractor reconstruction from time series data. The top panel shows how time series of three interacting "species" relate to the ecosystem attractor, using the Lorenz attractor as a hypothetical example. Bottom panel shows how a topologically equivalent shadow of the real system attractor can be reconstructed from the time series of a single species, by delay embedding its time series on itself. The Lorenz system typifies a time series that has large swings in state within a single attractor.

ecosystem according to the dynamics of a previous regime may result in further deterioration of ecosystem state, or even further regime shifts. Growing awareness of the importance of early detection of regime shifts, soon after they have occurred, has led to increased effort in developing methods to detect them (Scheffer et al. 2001, Rodionov 2005, Andersen et al. 2009). Parallel efforts have considered methods to predict regime shifts prior to their occurrence (as distinct from detection after a shift has occurred; e.g., Biggs et al. 2009, Dakos et al. 2012, Kéfi et al. 2014, Scheffer et al. 2015); here we focus on the detection aspect only.

Most methods currently used to detect regime shifts only describe change in ecosystem state, using time series data to identify sustained shifts in state (Biggs et al. 2012, but see Habeeb et al. 2005, Johnson 2009). These methods rely on having time series of multiple ecosystem variables from both before and after the suspected transition, to allow clear identification of system state both before and after the shift, and to distinguish sustained shifts in mean ecosystem state from stochastic fluctuations away from a steady state or deterministic cycles (Beaugrand et al. 2002, Mantua 2004, Rodionov 2004, Rodionov and Overland 2005). These approaches can identify significant change in ecosystems where sufficient long time-series data exist (e.g., Möllmann and Diekmann 2012, Beaugrand et al. 2015, Reid et al. 2015), but they have limitations. Firstly, the requirement for long time series data of multiple variables from both before and after the transition delays possible detection of regime shifts until long after they have occurred (as opposed to soon after they occur). Secondly, the data requirements are prohibitive for many ecosystems. Finally, because these methods track ecosystem state over time, any interpretation of changes in regime will be ambiguous since there could be sustained changes in state within the same regime (Di Lorenzo and Ohman 2013, Doney and Sailley 2013). The goal is therefore to develop capacity to distinguish changes in ecosystem state within the same regime or "basin of attraction," from a regime shift representing change to a different system attractor (Johnson 2009, Johnson et al. 2017).

An attractor is the region of multivariate phase space (values of state variables) to which a system (ecological system) of interacting entities (species) tends to return. A regime shift involves the transition of the system to a different attractor, with a consequent change in the structure and functioning of the system (Scheffer et al. 2001, Biggs et al. 2012). Transitions between attractors can occur when the ecosystem is pushed from one basin of attraction to another in response to pulsed or sustained perturbations, and can also relate to a change in stability of an attractor (Scheffer et al. 2001). The behavior of the system as a whole may 


\section{Box 1. Glossary of key terminology used in this paper.}

Attractor: A point or set of points in phase space to which a system is attracted, and to which it will tend to return following a perturbation.

Attractor reconstruction: Production of a representation of an attractor in phase space that is topologically equivalent to the real system attractor. The most common approach to attractor reconstruction is delay embedding.

Characteristic Length Scale (CLS): The optimal spatial scale at which to observe a system to obtain meaningful information on its behavior. The CLS is defined as the spatial scale at which stochastic and deterministic variation are balanced (Pascual and Levin 1999) and the scale above which windows in different regions of a landscape are statistically independent (Keeling et al. 1997). This scale emerges from the dynamics of the system and as such, a change in the CLS of a system is an indication that the dynamics of the system have fundamentally changed. Estimating the CLS involves attractor reconstruction combined with nearest neighbor prediction.

Delay embedding: A method of generating a multidimensional data set from an original data set in a single dimension. This is usually done with temporal data, but here in the sliding window approach to CLS estimation, we use spatial data. Each column in the resultant matrix will be one dimension, and each row one delay embedded point, in the reconstructed attractor in phase space.

Lorenz attractor: The Lorenz attractor (also called the butterfly attractor) is a based on a set of ordinary differential equations that describe and simplify atmospheric convection. We use it as a visual aid because it is a readily recognizable example of an attractor with complex (chaotic) dynamics (Figs. 1 and 2; Appendix S1). It also typifies a system that experiences large sustained swings in mean system state within a single regime.

Nearest neighbours: Nearest neighbours in a reconstructed attractor are a set of delay embedded points that represent similar local trajectories in time or space of the original time series or spatial series. Nearest neighbours are adjacent in the phase space, but not necessarily proximate in the original data series (see Fig. 2c).

Phase space: A space in which all possible states of a system are represented. Each state variable corresponds to one axis. Each point in phase space corresponds to a different ecosystem state.

Regime: An ecosystem regime constitutes the dynamic behavior and functioning of a system determined by its structure and self-supporting processes and feedbacks. Because it is dynamic in nature, a system can transition through many different states within the same ecosystem regime. An ecosystem regime is associated with a single attractor.

Regime shift: A change in the structure and functioning of an ecosystem (or other complex system) involving a switch to a different attractor. Also called phase shift or phase transition

State: An ecosystem state is the configuration of an ecosystem at one point in time (e.g., combination of population sizes, values of state variables). Importantly, there may be many possible states within an attractor, and even sustained and/or highly oscillatory changes in state (e.g., see time series in Fig. 1).

Variance spectra: plots of error variance (Error $X$ or Prediction $r^{2}$ ) against window size. This is also referred to as a fluctuation diagram in Keeling et al. (1997).

differ around different attractors. For example, a system may remain fairly stable, it may oscillate around an attractor (cyclical changes in ecosystem state), or it may move around the attractor in a haphazard manner in the face of multiple pulsed forcing events (Scheffer and Carpenter 2003, Scheffer et al. 2012). This complicates the detection of regime shifts and the characterization of observed change as regime shifts, and emphasizes that ecosystem state alone is insufficient to detect regime shift from one attractor to another.

Developing methods based on consideration of ecosystem attractors rather than ecosystem state provides a more objective means to detect regime shifts. Takens (1981) showed that it is possible to reconstruct the attractor of a system of interacting components from the time series of a single component of that system. This is based on the theory that the behavior of any one component is related to the behavior of the rest of the system and thus information about the system should be contained in the history (time series) of that component's behavior (Fig. 1). This is important because if the attractor of an ecosystem can be reconstructed, then it becomes possible to detect when the system shifts to an alternative attractor. This makes it possible to distinguish cases where changes in ecosystem state are due to oscillations around a single attractor, from cases where a change in system state reflects transition from one attractor to another. However, a key issue is that most methods of attractor reconstruction require very long, continuous time-series data, making them impractical for detecting change from one point in time to another.

Habeeb et al. (2005) provided a solution to the problem of unrealistic data demands by largely substituting space for time to reconstruct the system attractor, which they used to estimate the characteristic length scale (CLS) of ecological systems. The concept of CLSs was originally developed to determine the optimal spatial scale at which to observe the deterministic dynamics of an ecosystem, which is the scale of observation at which the signal to noise ratio is maximal (Keeling et al. 1997, Pascual and Levin 1999). Importantly, if a system shifts from one domain of attraction to another, i.e., there is a fundamental shift in space-time dynamics, then the shift is reflected as a change in the CLS (Johnson 2009). Conversely, there is no change in the length scale when the fluctuations in system state are within a single attractor even though the fluctuations may be large (Johnson 2009). Applications to real (marine) ecosystems show that CLSs can be calculated by considering space-time dynamics at the habitat (Habeeb et al. 2007) or species (Johnson et al. 2017) level and that, as the theory predicts, the CLS of the system can be estimated from attractor reconstruction based on the 
dynamics of a single component habitat or species, provided that components are connected directly or indirectly in an interaction network. However, a limitation of the approach is that to date it has only been applied using highly resolved two-dimensional spatial data (Habeeb et al. 2005, 2007, Johnson 2009, Johnson et al. 2017), which can be difficult to obtain for some ecological systems.

One-dimensional spatial data (transects) are collected far more commonly for a wide range of ecosystem types (e.g., grasslands, forests, reefs), and in marine systems is the form of data streams that emanate from autonomous underwater vehicles (AUVs), towed instruments, and acoustic information collected along ship tracks. Thus, adapting the CLS method to estimate characteristic length scales (and changes in these scales) using one-dimensional transect data would constitute a significant step forward in terms of detecting regime shifts in a much broader range of ecosystem types. This paper aims to (1) test whether the methods described by Habeeb et al. (2005) can be adapted to estimate the CLS of ecological systems using transect data; (2) use model systems to explore the types of ecological changes that can be detected as a change in the CLS; and (3) test the utility of the method applied to data obtained from one-dimensional transects on coral reefs in Indonesia.

\section{Methods}

The CLS of an ecosystem is the emergent spatial scale that arises from the dynamics between interacting species (see Box 1 for definition). Characteristic Length Scale estimation involves reconstruction of the system attractor by delay embedding spatial data, then using nearest neighbor trajectories (see Box 1) to predict subsequent points in the data series (Keeling et al. 1997, Pascual and Levin 1999), similar to the methods introduced by Sugihara and May (1990) for purely temporal data. Habeeb et al. (2005) adapted the long timeseries approaches of Keeling et al. (1997) and Pascual and Levin (1999) by substituting variation in space for variation in time to estimate the CLS of a system from a two-dimensional landscape based on either a single time step ("sliding window" method) or very few (4) time steps ("short time series" method). Here we describe how these two methods can be applied to one-dimensional transect data. Application of these approaches to a system assumes that the system has underlying deterministic dynamics resulting in emergent spatial pattern, and that spatial data can be obtained from the system. For more detailed information about the techniques described here, please refer to Kantz and Schreiber (2003).

\section{CLS estimation process}

The sliding window and short time-series approaches to CLS estimation primarily differ in how the delay embedding is constructed. We first describe the steps in detail in relation to the sliding window approach, following Fig. 2. Then we give an overview of the short time-series approach, highlighting how the steps differ in that approach.

Sliding window approach: prediction in space.-The "sliding window" approach can be used to estimate the CLS of a system from purely spatial data in a single time step (Fig. 2). In our case the base data is a transect of species inhabitancy recorded over regularly spaced intervals. Here we use presence-absence data, but note that the method could also work with count or concentration data.

1. Generate species densities.-From the transect data, we need to create series of species densities in one-dimensional windows of different sizes (with side length $l$ ). The minimum window size has a length that is two times the spatial resolution of the transect (e.g., if resolution is $1 \mathrm{~cm}$, the minimum window size will be $2 \mathrm{~cm}$ ). The choice of maximum window size is a trade-off between being large enough to capture the CLS, and small enough to achieve sufficient replication along the transect.

The species density series are generated by sliding windows of observation along the transect. A new vector of species densities is created for each window size. The window of observation slides along the transect by one-half the window length to each new window position ( $p$; Fig. 2a). The density $(X)$ of the target species is then calculated for each window position.

2. Attractor reconstruction.-The system attractor is reconstructed multiple times by delay embedding each vector of species densities generated for each window length $(l)$ into $d_{\mathrm{E}}$ embedding dimensions. For the sliding window approach, the delay $(\tau)$ is a spatial delay. Habeeb et al. (2005) found that, for the sliding window method, a proportional delay works better than a fixed delay, such that $\tau=\alpha \times$ window length. We found 1D CLS estimates to be robust to choices of $\alpha$, and here we set $\alpha$ to $50 \%$ of the window length, as described in 1. Generate species densities. One representation of the attractor is reconstructed per window size by delay embedding the vector of densities generated for that window size. To do this, a matrix is constructed where the original vector of species densities forms the first column. The second column is the same vector of densities, delayed by one spatial step (minus the density in the initial window; see Fig. 2b for illustration of delay embedding). Each column of the resultant matrix is one dimension of the reconstructed attractor, and each row is a single delay embedded point. Each embedded point therefore captures the trajectory of the species density forward in space by $d_{\mathrm{E}}$ number of window positions. We found 1D CLS estimates to be robust to choice of $d_{\mathrm{E}}$, and here use a $d_{\mathrm{E}}$ of 3 .

In theory, observations of any of the interacting species in a system can be used to reconstruct the system attractor (Abarbanel 1996), though Habeeb et al. (2005) found some sensitivity to choice of species when estimating the CLS from long time series. We use observations of a number of species to reconstruct separate representations of the attractor of each system.

3. Prediction.-Many more small windows fit along the transect than large windows so there will be many more points in the attractor reconstructed from small windows than those reconstructed with large windows. To avoid resultant bias in predictive power, $N$ equally sized samples (we used $N=20$ ) of delay embedded points are generated for each window size by randomly sampling with replacement from the delay embedding of each window length.

The aim is to predict the next point in space (which is effectively the $d_{\mathrm{E}}+$ first dimension from the reconstructed attractor). To do this, $k$ nearest neighboring points to each sample 


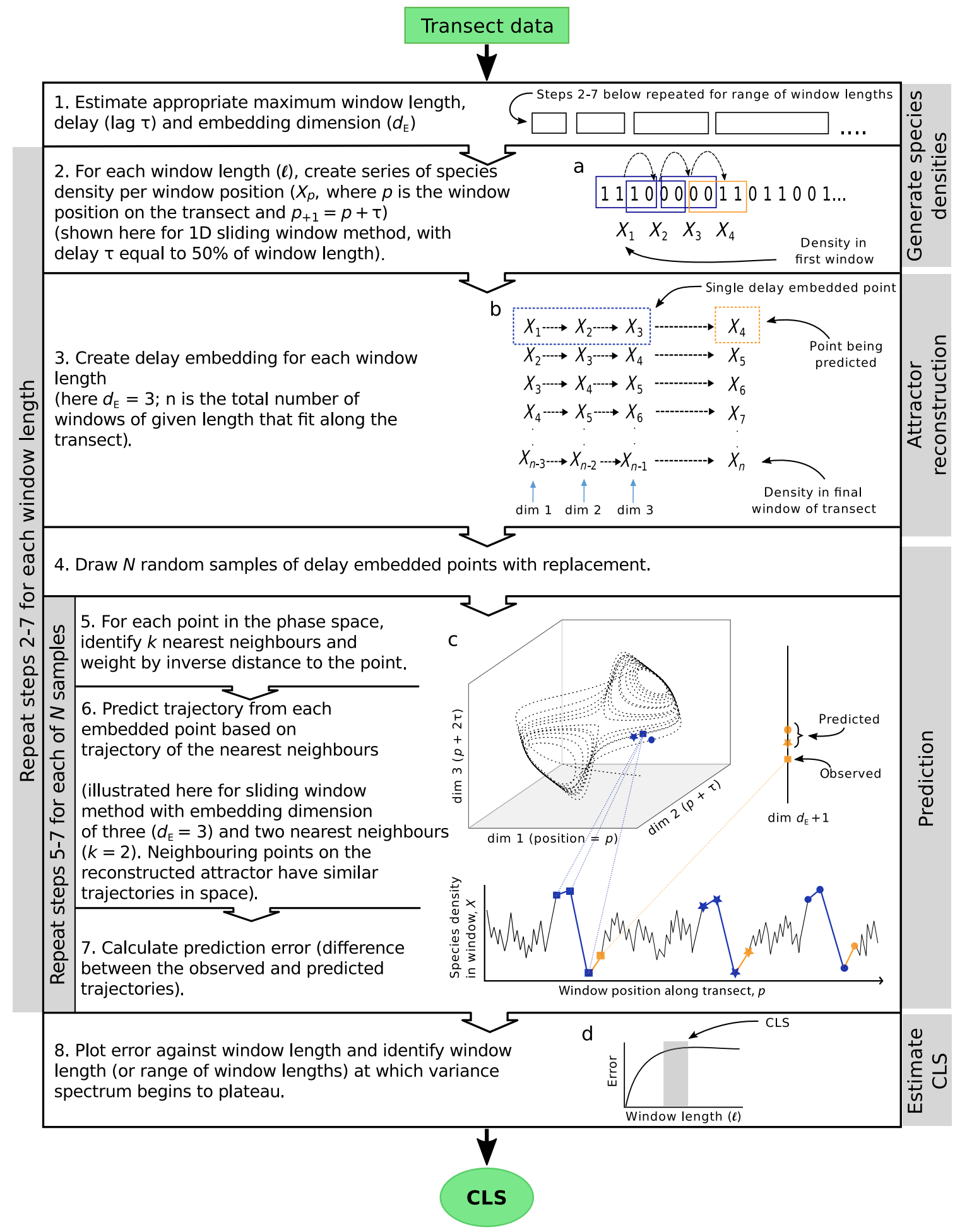

FIG. 2. Flow diagram showing the steps in the characteristic length scale (CLS) estimation method. Panels a-c illustrate the process for the one-dimensional sliding window approach. A similar diagram is provided for the one-dimensional short time-series approach in Appendix S1. For more detailed descriptions of the techniques, see Kantz and Schreiber (2003). (a) Slide the 1D window of observation along the transect and calculate mean number (density) of the target species in each window $(0=$ absent, $1=$ present $)$. (b) Delay embed the spatial data series so that the trajectory over $d_{\mathrm{E}}$ sequential windows becomes a single point on the reconstructed attractor (shown in panel c). (c) Identify $k$ nearest neighbours of each point in the reconstructed attractor space and predict density $X$ in the subsequent window based on the trajectory of neighboring points. Here, the blue square represents the three-dimensional point of interest in the attractor space, and the star and circle its nearest neighbours. The orange square is the point being predicted, based on the orange star and circle. The lower plot illustrates how each $d_{\mathrm{E}}$ dimensional point in the reconstructed attractor maps to a $d_{\mathrm{E}}$ trajectory of densities in space, and that nearest neighbours in the attractor space have similar trajectories in space but are not neighboring in space. (d) The characteristic length scale (CLS) is the window length at which prediction error begins to plateau. 
point in the attractor space are located using the $\mathrm{nn} 2$ function from the RANN package (Arya et al. 2017) in R (R Core Team 2017). The trajectory from each embedded point is predicted from the trajectories of the nearest neighbours (see definition in Box 1), with the prediction being weighted toward the trajectories of the closest neighbours (inverse distance weighting) (Fig. 2c). To illustrate, in Fig. 2c, the blue square on the reconstructed attractor is the delay embedding of the three blue squares (representing species densities) in the spatial series in the panel below. The point to be predicted is the species density in the following window position (the orange square). The two nearest neighbours are represented by a blue star and circle in the reconstructed attractor, and represent similar sequences of species density in space. The trajectories from the two nearest neighbours (the orange star and the circle on the lower panel) are used to predict the next species density (the value of the orange square). To calculate the prediction error, the predicted value (the weighted average of the orange star and circle) is then compared with the observed species density (the value of the orange square). Initial testing showed that 1D CLS estimates were robust to the choice of $k$ (we set $k$ equal to 7).

There are two available measures of prediction error: Error $X$ (Keeling et al. 1997) and Prediction $r^{2}$ (Pascual and Levin 1999). Previous work (Trebilco 2002) showed that Error $X$ produces the most interpretable curves when using the sliding window method (but that Prediction $r^{2}$ was the better metric when using the short time series approach), and our initial testing supported that finding. We therefore use Error $X$ as the metric to identify the CLS using the sliding window method, but present examples of both metrics.

A. Error $X$.-For two-dimensional data, Error $X$ is calculated as follows:

$$
\text { Error } X=l \sqrt{E\left[\left(X_{l}^{p}-\hat{X}_{l}^{p}\right)^{2}\right]}
$$

where $X_{l}^{p}$ is the observed and $\hat{X}_{l}^{p}$ is the predicted density of species $X$ in a window of length $l$ at window position $p$, and $E$ is the expectation of their difference for that window length (mean difference over different window positions).

For one-dimensional data, a window of length $l$ contains less information than a two-dimensional window of the same length because the area contained in the two-dimensional window is $l^{2}$. Thus for one-dimensional data, Error $X$ is given by

$$
\text { Error } X=\sqrt{l E\left[\left(X_{l}^{p}-\hat{X}_{l}^{p}\right)^{2}\right]}
$$

B. Prediction $r^{2}$.- The Prediction $r^{2}$ metric of Pascual and Levin (1999) is given by

$$
\text { Prediction } r^{2}=1-\frac{E\left[\left(X_{l}^{p}-\hat{X}_{l}^{p}\right)^{2}\right]}{\operatorname{Var}\left(X_{l}^{p}\right)}
$$

4. Estimate the CLS. - Finally, the prediction errors are plotted against window length, to produce a variance spectrum showing how prediction error varies with the scale of observation (Fig. 2d). For both Error $X$ and Prediction $r^{2}$, the CLS is defined as the window size at which the variance spectra begin to plateau (following Habeeb et al. 2005, Johnson 2009). In practice, the precise window size at which the curve begins to plateau can be difficult to pinpoint. Accordingly, the CLS is given as a range of possible window sizes.

Short time-series approach: prediction in time.-This approach to CLS estimation involves reconstructing the system attractor from short-time series of observations from multiple window positions placed along the transect (see Appendix S1 for figures illustrating this approach). As in the sliding window approach, each window is placed one-half a window length along the transect from the previous window. The density of a given species in each window position is then tracked for a small number of time steps (four or five), where each time step acts as one dimension of the embedding and the final time step is the one being predicted. Unlike in the sliding window approach above, this delay $\tau$ is a temporal lag. We use a delay of ten time steps, as per Habeeb et al. (2005) and Johnson (2009). The attractor of the system is then reconstructed for each window size from the multiple short time series. Then, for each point, the density in the next time step is predicted from the trajectories of $k$ neighboring points on the attractor. This is the key difference between the two approaches: in the short time-series method the density in the same window position in the following time step is being predicted, whereas in the sliding window method the density the next window position in space (in the same time step) is being predicted.

\section{Detection of regime shifts}

Regime shifts can be detected by comparing the CLS of the system from time to another. A change in the CLS of a system from one time to another indicates that there has been a regime shift (Johnson 2009, Johnson et al. 2017). When the CLS of a system is identified, it is given as a range of possible window sizes (see CLS estimation process). A regime shift can only be detected when the CLS range in one time step does not overlap the CLS range from the other time step.

\section{Model test systems}

To create test cases, we planned to run ecosystem models for 5,000 time steps, change the model parameters to simulate different types of ecosystem change, then continue the model run for a further 5,000 time steps. The test systems we used are two-dimensional toroidal probabilistic cellular automata of interacting species, programmed in Python (version 3.5.2; Python Software Foundation, http://www.python.org/). In the model simulations, the initial cover of species over the model landscape is set by randomly selecting a species to inhabit each cell (pixel) within a 2,000 × 6,000-pixel landscape. Species interactions are governed by a set of overgrowth probabilities, where each species has a set probability of overgrowing each other species. At each time step, the species in each cell interacts with one randomly selected neighboring cell. To determine the outcome of each interaction, a random number between 0 and 1 is generated. If the random number is less than the probability that the cell would be 
overgrown by the species in the neighboring cell, then the cell is replaced by the species in the neighboring cell when the landscape is updated in the next the step. Otherwise, the species inhabiting the cell continues to inhabit that cell into the next time step. To avoid artefacts of sequentially updating the landscape, after each individual cell interacts with one neighbor at time $t$ the result is written to a "new" working landscape matrix representing time $t+1$, and once the whole landscape is updated the new landscape at $t+1$ replaces the landscape at $t$ before the next new time step begins. The competitive dynamics of each system and the changes to those dynamics are described in test cases of ecosystem change.

Test cases of ecosystem change.-To evaluate whether the 1D CLS estimation methods could detect a change in system dynamics, we used the test cases previously described by Johnson (2009). Johnson's model systems were run in the Compete software package (Johnson 2002) (and analysed using the twodimensional CLS methods) (Johnson 2009). The simulations in this paper were run in Python to be able to consider larger landscapes (from which long transects could be extracted) than is possible with Compete. A summary of test cases, species considered, metrics and figures presented is provided in Table 1.

Four test cases based on two model systems were considered. The first model system is a five-species intransitive loop, where each species is able to overgrow just one other species, and does so in $100 \%$ of occasions in which they interact. This system is structured so that Species 1 overgrows Species 2, which overgrows Species 3 and so on: Species $1>$ Species $2>$ Species $3>$ Species $4>$ Species $5>$ Species 1. After this model system runs for 5,000 time steps, the system dynamics change and the model runs for a further 5,000 time steps (continuing from the final landscape of the previous "regime"). The change in dynamics brought about in this second regime were as follows:

1) Test case 1: A change in the environment results in the growth rate of Species 5 being reduced to $75 \%$ of its original growth rate. This is achieved by reducing the probability that it overgrows Species 1 from $100 \%$ to $75 \%$ of occasions in which they interact.

2) Test case 2: A change in the environment causes a change in the way Species 1 and Species 2 interact, such that Species 1 now only over grows Species 2 in $60 \%$ of interactions and Species 2 overgrows Species 1 in the other $40 \%$ of occasions in which they interact.

In the second set of test cases, the initial system is a 20species system interacting according to a random interaction matrix. The system initially has open recruitment, meaning that no species can become permanently extinct from the landscape, regardless of how poorly competitive it might be. At each time step, $0.0001 \%$ of cells suffer random mortality (disturbance) events, so that those cells become empty space in the following time step. Any of the species are then able to recruit to one of these empty cells, where each species has a $1 \%$ chance of being recruited to each empty cell at each time step. After 5,000 time steps, the dynamics change as follows:

3) Test case 3: The system changes such that open recruitment stops. According to these new rules, empty space can only be filled by the overgrowth of a species from one of the four neighboring cells.

4) Test case 4: An invasive species arrives. Open recruitment is maintained; however, a 21st species of a highly invasive nature enters the system. This invader overgrows three randomly selected species in $80 \%$ of occasions in which they interact, and is overgrown by those same species in the other $20 \%$ of interactions. Interactions between the invader and any of the other 17 species result in standoff, i.e., it neither overgrows, nor is overgrown by, any of those 17 species.

For each test case, we chose to present the spectra estimated from three species. For Test cases 1 and 2, spectra estimated from Species 2, 3, and 4 are presented. For Test Cases 3 and 4 , three species were chosen to analyze based on their abundance in regime 1: Species 19 was the most abundant, Species 10 was the third most abundant, and Species 3 was the fifth most abundant. The spectra from Species 21 was also analysed and presented for Test Case 4, regime 2. For each test case, three transects were extracted from each of the final landscapes (of the first and second regimes) to test the sliding window method. We present the spectra of all three transects for each species together to demonstrate variability amongst transects. To test the short-time series method, transects were taken from four landscapes separated by ten time steps at the end of each regime for Test Cases 1-3. However for Test Case 4, transects for the short time-series method were taken from landscapes separated by 50 time steps because regime 2 of this test case evolved so slowly that there was very little difference between landscapes separated by only 10 time steps.

\section{Real world test case}

We applied the sliding window 1D CLS estimation method to pre-existing coral reef benthic community transect data from four sites within the Wakatobi Marine National Park, Sulawesi, Indonesia (Haapkylä et al. 2016). The collection of these data is described in Haapkylä et al. (2007, 2009, 2015, 2016). Briefly, three replicate line-intercept transects of $20 \mathrm{~m}$ length and $1 \mathrm{~cm}$ resolution were recorded from the slope, crest, and flat of each of these sites in 2007, 2010, and 2011. The three replicate transects at each site followed a fixed depth contour in each habitat, and were separated by $5 \mathrm{~m}$. When the $20 \mathrm{~m}$ coral reef replicate transects were analysed individually, the CLS sometimes appeared to be larger than the maximum window size we were able to consider. Accordingly, the coral reef transects from each site were treated as a single transect to maximize the maximum window size that could be considered. The maximum window size and transect length were kept consistent (100 $\mathrm{cm}$ and $60 \mathrm{~m}$, respectively) between sites to ensure CLSs were comparable.

Here we present the CLS estimated from each of the reef slopes (Blue Bowl, Coral Gardens, Sampela1 Reef, Hoga Buoy2 Reef), and discuss both temporal and spatial differences in the estimated CLSs within and among the sites. Initial testing of the short time-series method on this data set showed that this method was inappropriate for the data set, because of the nature of the data set and the number of time steps available (only three time steps). We therefore only estimated CLS using the sliding window approach. The CLSs presented for each reef are based on morphotypes rather than species. 


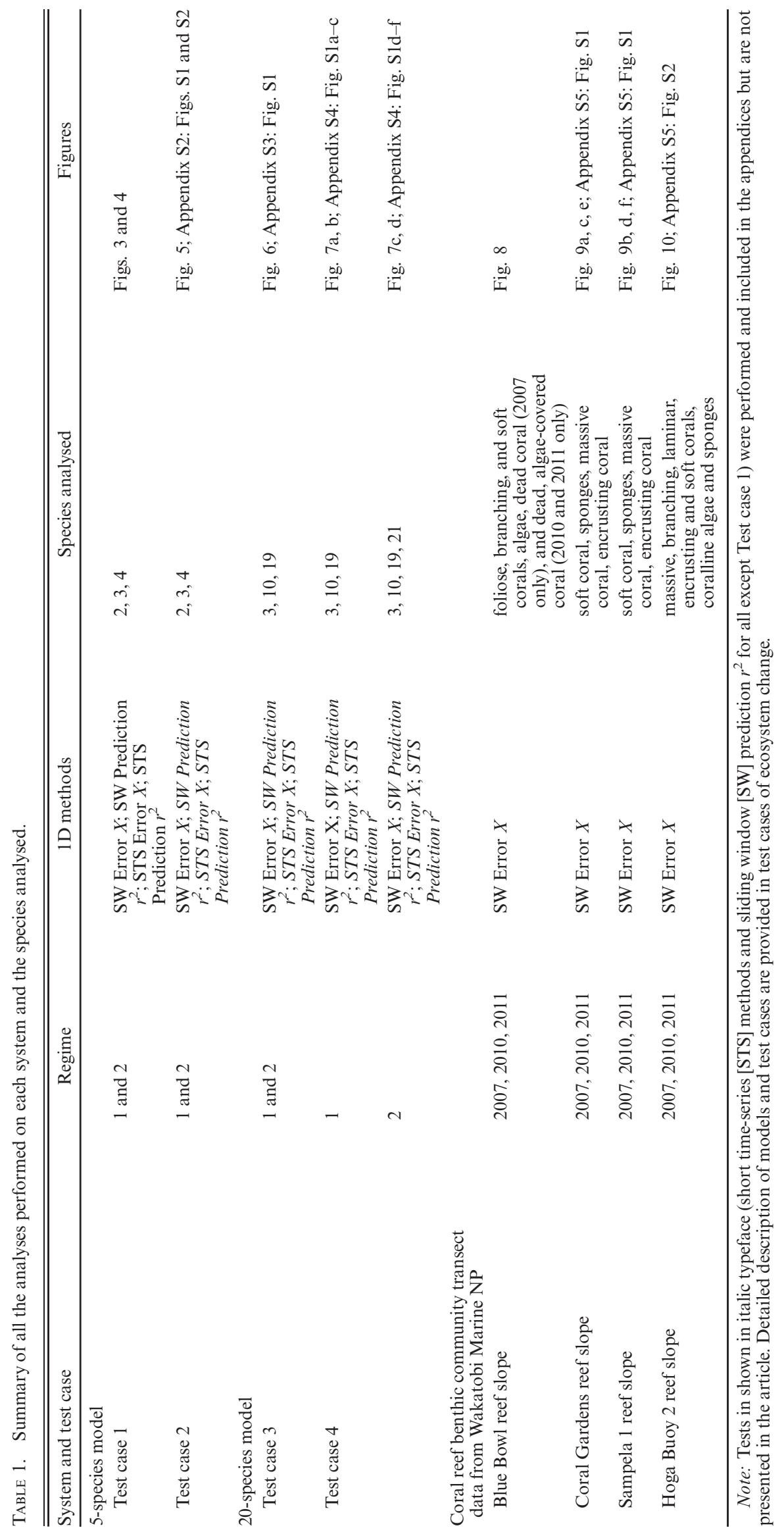



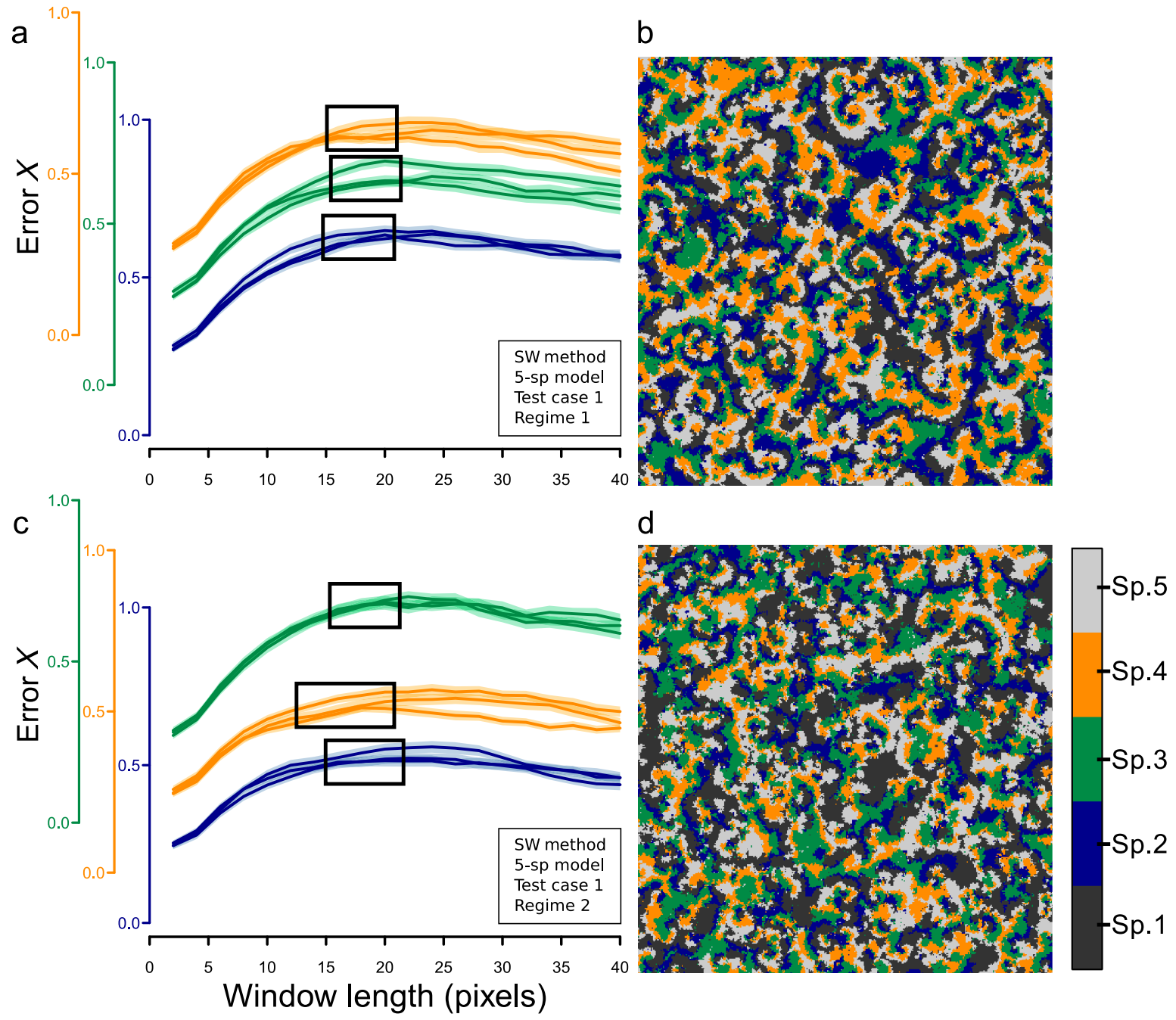

Fig. 3. Test case 1 Error $X$ spectra from the one-dimensional sliding window method for (a) regime 1 and (c) regime 2 and corresponding model landscape sections of $400 \times 400$ pixels from (b) regime 1 and (d) regime 2. Species are shown in the same colors in both the spectra and the landscapes as indicated by the color bar. For each of three species, spectra from three replicate transects are shown together to demonstrate variability due to transect position. Solid lines are a rolling mean (three windows) of the Error $X$ estimates from the 20 sets of random samples for each window size, and shading indicates the $95 \%$ pointwise confidence intervals. The black boxes indicate the CLS range estimated from each species. The reduction of the growth rate of Species 5 in regime 2 did not result in a change in the CLS in this test case.

Decisions on which morphotypes to investigate and present for each reef were based on whether morphotypes had sufficient coverage to produce an interpretable curve in each of the three years, so that comparisons could be made between years.

\section{Analyses}

All model test case simulations were run using the NumPy and Numba packages in Python (Python Software Foundation, version 3.5.2, available at http://www.python.org) Anaconda (Anaconda Software Distribution, version 4.2.0, https://anaconda.com) on a Ubuntu (16.04.1 LTS x86_64) system hosted by NeCTAR. NeCTAR is a cloud computing research environment supported by the Australian Government through the National Collaborative Research Infrastructure Strategy. Landscape data were exported in NumPy format and read into RStudio using the RcppCNPy library (version 0.2.6; Eddelbuettel and $\mathrm{Wu}$ 2016). Transects were sampled, data formatted, and analyses performed in the RStudio (version 1.1.383, RStudio Team 2015) environment and R (version 3.4.1, R Core Team 2017) using version 2.5.1 of the RANN package (Arya et al. 2017). All code for test case simulation and analysis is available as supplementary material in Data S1, and all coral reef transect data are available through Australian Ocean Data Network (Haapkylä et al. 2016). The 1D CLS estimation R package is available through GitHub (available online). ${ }^{6}$

\section{RESULTS}

\section{Model test cases}

Test case 1.-The estimated CLS of the first regime in Test case 1 was $15-20$ pixels. After the Species 5 growth rate was reduced, Species 1 increased in abundance, Species 2 and 4

\footnotetext{
${ }^{6}$ https://github.com/DelphiWard/CLS1D
} 
decreased in abundance, and the abundance of Species 3 and 5 increased very slightly. Despite these observed changes in abundance, there was no clear change in the estimated CLS between the two regimes, with the CLS in the second regime also being estimated at 15-20 pixels (Fig. 3). The lack of change in CLS indicates there was not a regime shift in this test case. Although the estimated CLS did not change, the shape of the sliding window Error $X$ spectra from Test case 1 differs between the two regimes. The main difference in the spectra is that while the magnitude of Error $X$ was equivalent between species in the first regime, the height of the curve (magnitude of Error $X$ ) for species 2 and 4 were smaller in the second regime, coinciding with declines in the numbers of those species observed within the landscape. Despite this, the emergent CLS of the system remained consistent when estimated from the different species, as expected from the theory, indicating that the species are indeed interacting within the same community (Rand and Wilson 1995, Keeling et al. 1997, Pascual and Levin 1999, Habeeb et al. 2005).

Prediction $r^{2}$ spectra proved more difficult to interpret than the Error $X$ spectra. The sliding window prediction $r^{2}$ spectra appear inverted (Fig. 4a, d), as had been previously reported by Trebilco (2002). Similarly, the CLS is not estimable from the short time-series prediction $r^{2}$ spectra for Test case 1 (Fig. 4c, f). These spectra continue to increase with window length and do not appear to reach a plateau. The short time-series Error $X$ spectra in both regimes for Test case 1 indicated a CLS of approximately 5-10 pixels, which is smaller than that of the sliding window spectra (Fig. 4b, e). Interestingly, these spectra exhibit a decline in Error $X$ after a peak is reached. Given the difficulty interpreting the 1D prediction $r^{2}$ spectra, and the ultimate goal for this method to be used to detect change between time steps, we hereafter only present sliding window Error $X$ spectra (the other spectra are available in the appendices).

Test case 2.-All three species considered in Test case 2 indicate a CLS of approximately 15-20 pixels in the first regime (Fig. 5a, b). After the interaction between Species 1 and Species 2 changed, the abundance of Species 1, 2 and 4 increased in the landscape, while Species 3 and 5 decreased (Fig. 5d). In this second regime, the CLS estimated from spatial patterns of Species 2 and 4 increased to 25-30 pixels (Fig. 5c). For Species 3, the CLS in the second regime was difficult to interpret (when considered on the same $y$-axis scale) because its Error $X$ curve was very shallow, seemingly a consequence of its low abundance. Nevertheless, the estimation of the CLS as 25-30 pixels was supported when the Error $X$ spectra was considered over a smaller $y$-axis range (Appendix S2: Fig. S2). The CLS of this system changed so we detected a regime shift in this test case.

Test case 3.- Species 10 and 19 indicated a CLS of around 20 pixels in the first regime of Test case 3, while the CLS
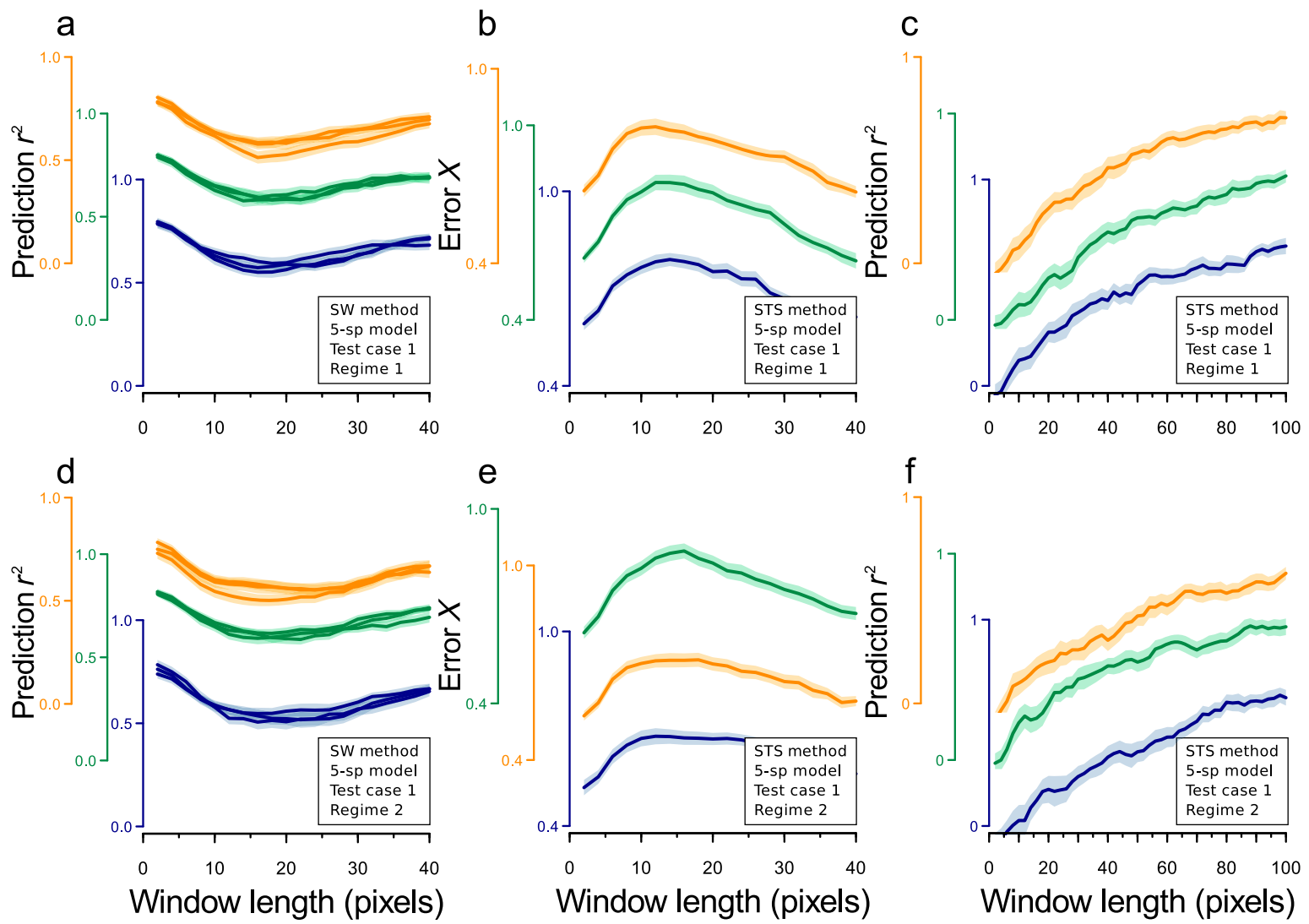

FIG. 4. Further Test case 1 spectra obtained from one-dimensional "transect" data. Sliding window (SW) prediction $r^{2}$ spectra for (a) regime 1 and (d) regime 2; short time series (STS) Error $X$ spectra for (b) regime 1 and (e) regime 2 and short time-series prediction $r^{2}$ spectra for (c) regime 1 and (f) regime 2. Solid lines are a rolling mean (three windows) of the Error $X$ estimates from the 20 sets of random samples for each window size, and shading indicates the $95 \%$ pointwise confidence intervals. Colors as per Fig. 3. 

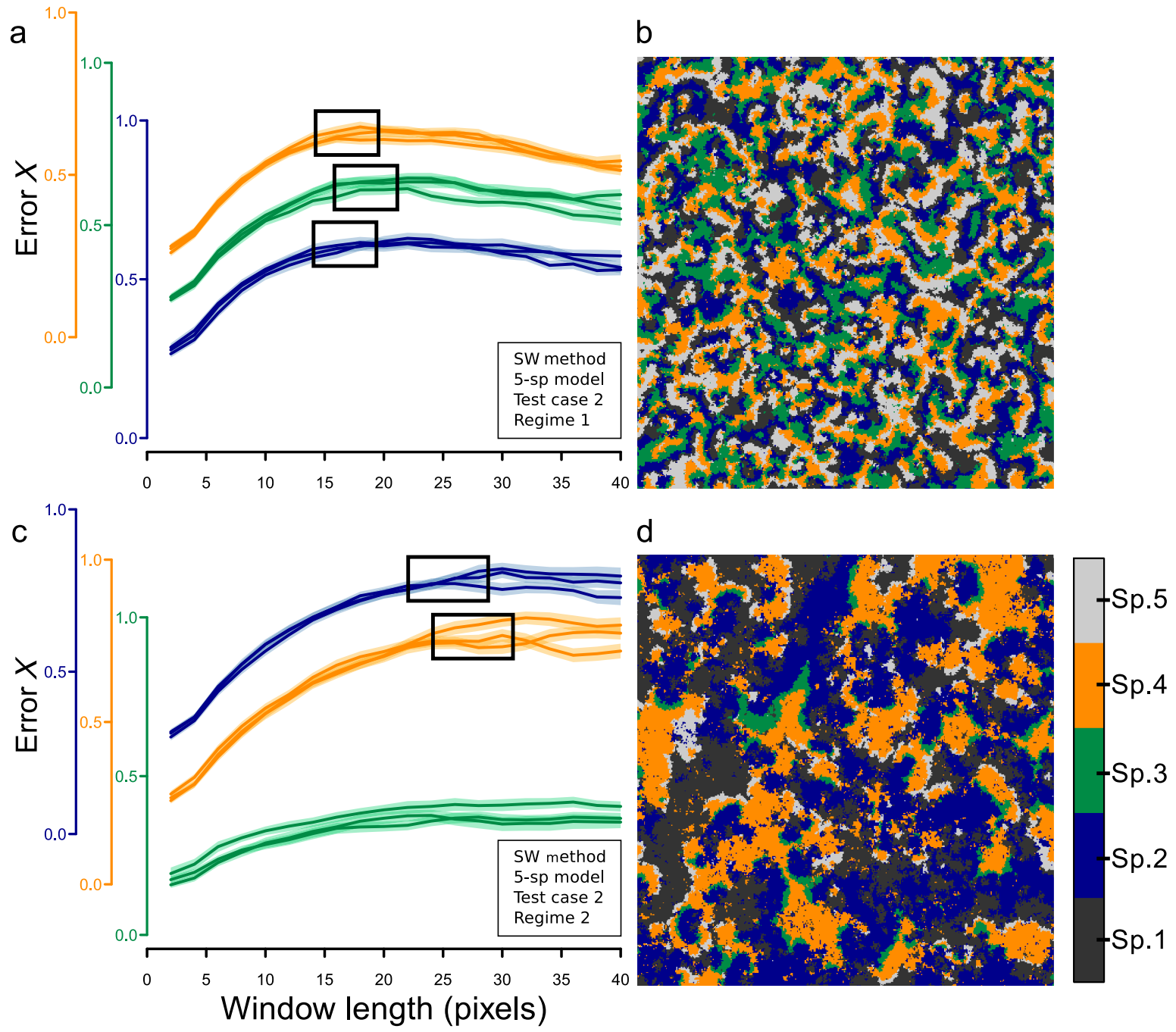

FIG. 5. Test case 2 Error $X$ spectra from the one-dimensional sliding window method for (a) regime 1 and (c) regime 2 and corresponding model landscape sections of $400 \times 400$ pixels from (b) regime 1 and (d) regime 2 . Species are shown in the same colors in both the spectra and the landscapes, as indicated by the color bar. For each species, spectra from three replicate transects are shown together to demonstrate variability due to transect position. Solid lines are a rolling mean (three windows) of the Error $X$ estimates from the 20 sets of random samples for each window size, and shading indicates the $95 \%$ pointwise confidence intervals. The black boxes indicate the CLS range estimated from each species. In this test case, the CLS increased from 15 to 20 pixels in the first regime, to 20-25 pixels in the second regime following a change in the interaction between Species 1 and 2. See Appendix S2 for spectra of Species 3 from regime 2 on a smaller $y$-axis scale.

indicated by Species 3 is larger: between 20 and 30 pixels (Fig. 6a, b). In the randomly determined interaction matrix, Species 3 typically had low probabilities of either overgrowing or being overgrown by other species, so its dynamics may be somewhat more independent than those of species with strong interaction probabilities. In the second regime, the CLS indicated by each species remains unchanged after the cessation of open recruitment, suggesting that scale of this system is dominated by interaction outcomes rather than by recruitment (Fig. 6c, d). Accordingly, we did not detect a regime shift in this test case.

Test case 4.-The CLS estimates from Species 10 and 19 in the first regime of Test case 4 are 15-20 pixels, similar to those of Test case 3 (Fig. 7a, b). Again in this test case, Species 3 indicates a slightly larger CLS, but the curves are more difficult to interpret, and are more variable between transects than for the other species. Following the invasion of a new, highly competitive Species 21 in the second regime, the landscape became dominated by that invasive species while the abundances of other species fell (Fig. 7d). This is reflected in much shallower Error $X$ spectra for Species 3 and 19 (Fig. 7c). For both of these species, the spectra became harder to interpret. Species 19 appears to indicate a CLS of $10-15$ pixels in regime 2 , and Species 3 possibly indicates a CLS of 20-25 pixels, though this is difficult to estimate. Neither Species 3 nor Species 19 interacted directly with Species 21. Species 10 was one of the three species that directly interacted with Species 21. Although Species 10 was still present in very low numbers on the final landscape, it was absent from all three transects and so Error $X$ could not be calculated from this species. In the final landscape, the 


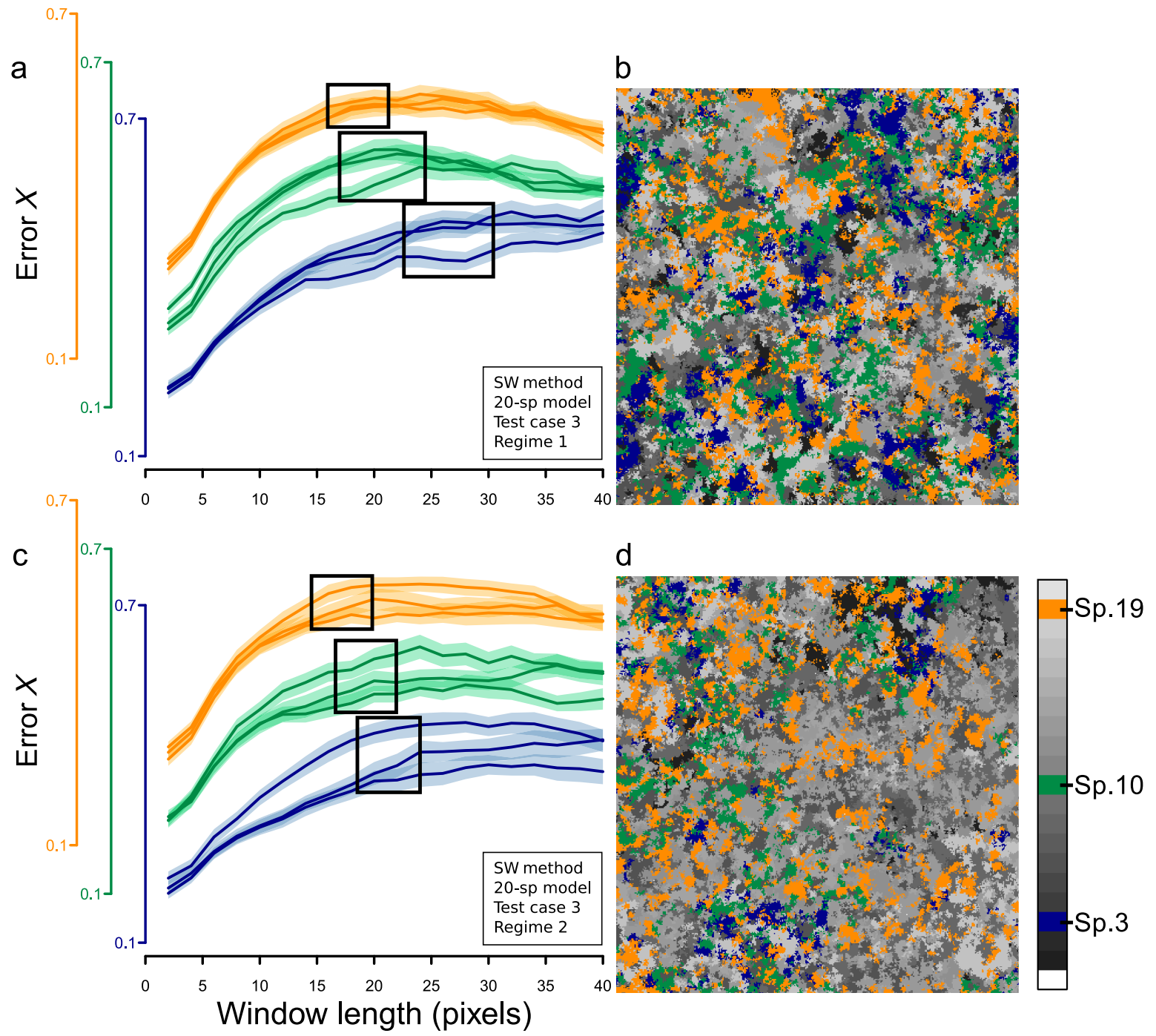

FIG. 6. Test case 3 Error $X$ spectra from the one-dimensional sliding window method for (a) regime 1 and (c) regime 2 and corresponding model landscape sections of $400 \times 400$ pixels from (b) regime 1 and (d) regime 2. Species are shown in the same colors in both the spectra and the landscapes, as indicated by the color bar. For each species, spectra from three replicate transects are shown together to demonstrate variability due to transect position. Solid lines are a rolling mean (3 windows) of the Error $X$ estimates from the 20 sets of random samples for each window size, and shading indicates the $95 \%$ pointwise confidence intervals. The black boxes indicate the CLS range estimated from each species. There was no change in the CLS following the cessation of open recruitment in this test case.

Species 21 spectra indicate a clear CLS of 10-15 pixels. The changes in CLS observed in this test case indicate that there was a regime shift.

\section{Coral reefs}

Blue Bowl reef.-Blue Bowl reef exhibited large changes in CLS over the time period 2007 to 2011 (Fig. 8, Table 2). In 2007 , this reef was dominated by foliose coral, which indicated a CLS of the order of 55-70 cm. Other morphotypes considered were branching coral, which indicated a CLS of about $65 \mathrm{~cm}$, soft coral showing a CLS of about $45 \mathrm{~cm}$, and algae, which indicated a CLS of about $25-30 \mathrm{~cm}$. These length scales had changed markedly in 2010. In 2010, the CLS indicated by foliose and branching corals had declined to $30-35 \mathrm{~cm}$, and that indicated by soft coral had declined to around $35 \mathrm{~cm}$. The CLS determined from cover of algae is harder to distinguish in 2010, but appears to be around $45 \mathrm{~cm}$. Interestingly, the CLS of dead, algae-covered-coral was about $60 \mathrm{~cm}$ in 2010, similar to that of foliose coral and dead coral in 2007 (though with a much higher abundance than the latter). This probably reflects the spatial patterns of (now deceased and overgrown) foliose coral. In 2011 the CLSs were largely similar to those of 2010. The CLS indicated by cover of foliose coral was $35-50 \mathrm{~cm}$, while that estimated from branching coral appeared to increase slightly to $40-55 \mathrm{~cm}$. CLSs determined from cover of algae and dead, algae-covered-coral remained unchanged at $40-55 \mathrm{~cm}$ and $60 \mathrm{~cm}$ respectively. The changes in CLS on this reef indicate that a regime shift occurred between 2007 and 2010 and that the new regime persisted into 2011.

Coral Gardens.-For the most part, the CLSs determined from the cover of morphotypes on the Coral Gardens reef 


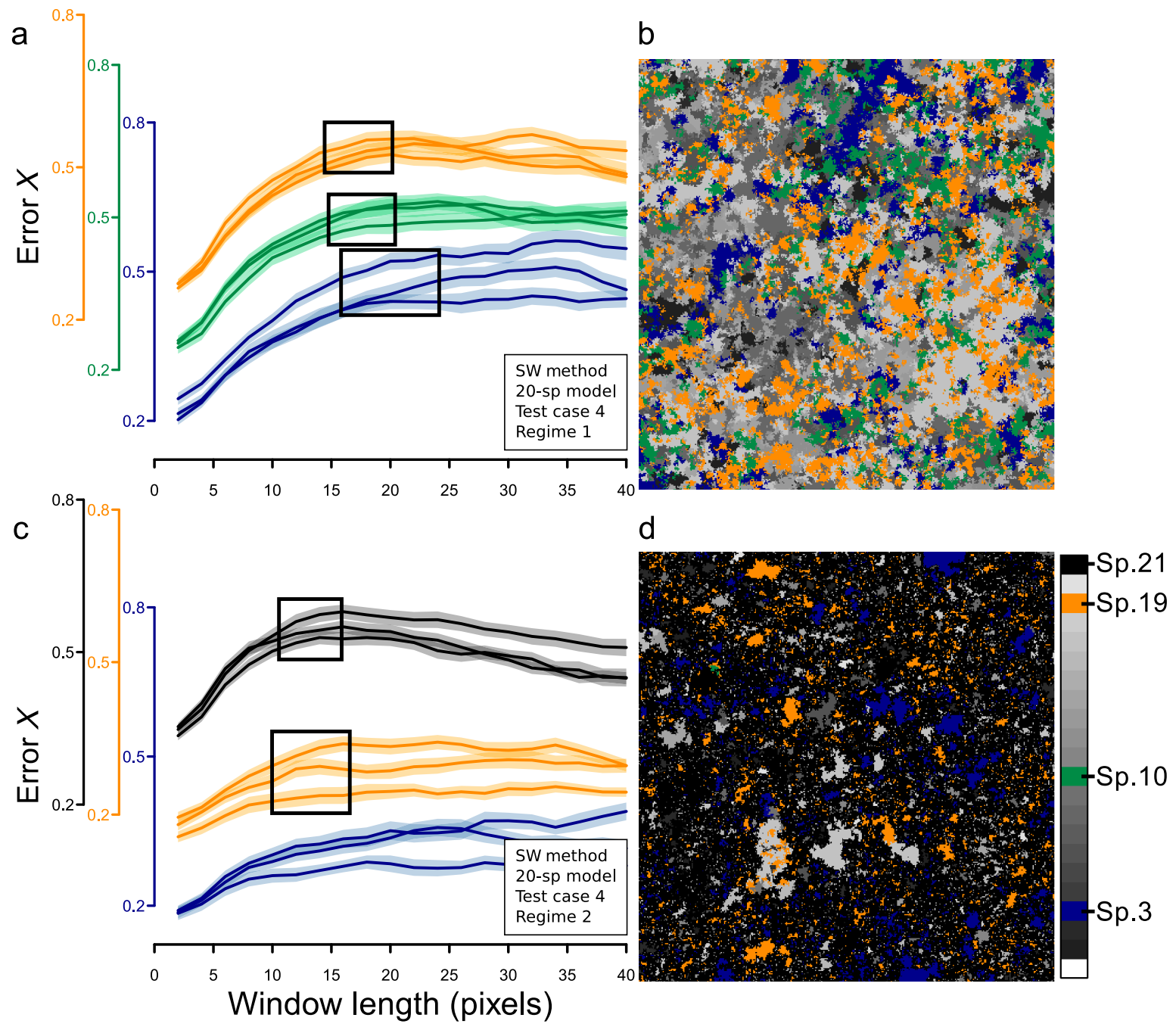

FIG. 7. Test case 4 Error $X$ spectra from the one-dimensional sliding window method for (a) regime 1 and (c) regime 2 and corresponding model landscape sections of $400 \times 400$ pixels from (b) regime 1 and (d) regime 2. Species are shown in the same colors in both the spectra and the landscapes; the invasive species is Sp.21 in black. For each species, spectra from three replicate transects are shown together to demonstrate variability due to transect position. Solid lines are a rolling mean (three windows) of the Error $X$ estimates from the 20 sets of random samples for each window size, and shading indicates the $95 \%$ pointwise confidence intervals. The black boxes indicate the CLS range estimated from each species. In this test case, there was a decline in CLS from 15 to 20 pixels in regime 1 to 10-15 pixels in regime 2 following the invasion of a new species to the landscape.

slope remained unchanged between 2007 and 2011 (Fig. 9a, c, e, Table 3). Coral Gardens was dominated in coverage by soft coral, and in 2007 the CLS of this morphotype was 60-80 cm. In 2007, encrusting coral and sponges both indicated a CLS of about $30 \mathrm{~cm}$, while that derived from branching coral was slightly larger. In 2010, the CLSs indicated from the latter three morphotypes were largely unchanged, but that shown by soft coral had declined. The spectra for encrusting coral in 2010 appears shallower, despite having similar cover and CLS in both years. In 2011, the CLS indicated by encrusting coral had declined slightly, but soft coral indicated a similar CLS to 2007. Sponges indicated a similar CLS to the previous years. The CLS was difficult to estimate from branching coral for 2011, but was possibly around $35 \mathrm{~cm}$. The lack of clear changes in CLS mean that there is no evidence of a regime shift at this reef.
Sampelal reef.-Sampela reef slope only exhibited small changes in CLS between 2007 and 2011, despite experiencing declines in cover of all morphotypes considered except encrusting coral (Fig. 9b, d, f, Table 4). In 2007 the CLS indicated by massive coral, soft coral, and sponges was around 50 to $60 \mathrm{~cm}$. The CLS was harder to determine from encrusting coral, but appeared to be about $40 \mathrm{~cm}$. In 2010, the CLS indicated by encrusting coral was slightly smaller, at around $35 \mathrm{~cm}$, but that shown by the other morphotypes was either unchanged (sponges) or had declined (soft coral and massive coral). The CLS ranges estimated from soft coral do not overlap between 2007 and 2010; however the CLS range estimated in 2011 overlaps both the previous years. Between 2010 and 2011 there was no change in CLS estimates from encrusting coral or massive coral, but there was a slight increase in that indicated by soft coral, and there was a decline in the CLS indicated by sponges to $25-45 \mathrm{~cm}$. 

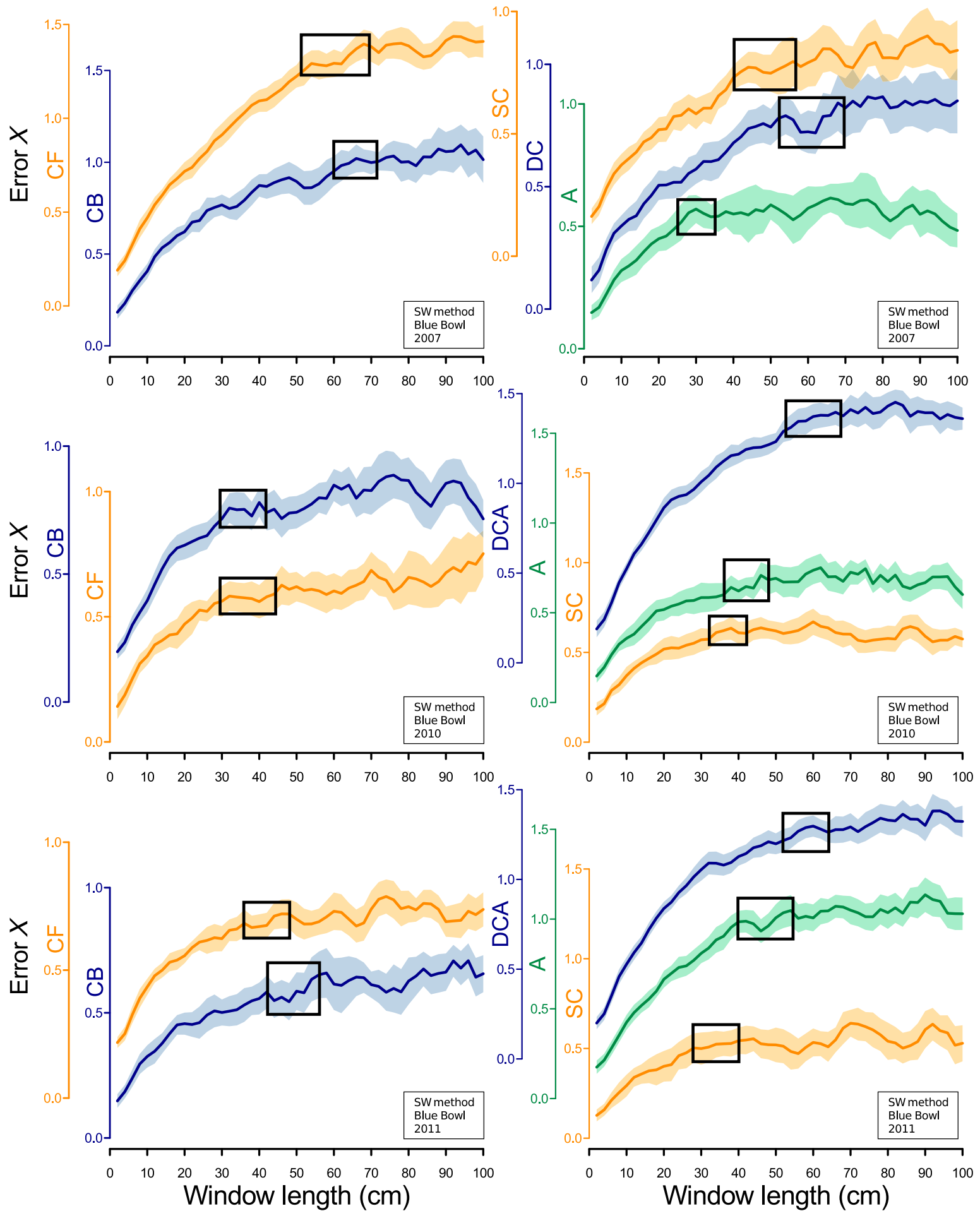

FIG. 8. Error $X$ spectra determined from cover of five morphotypes from Blue Bowl reef in 2007, 2010, 2011. Solid lines are a rolling mean (three windows) of the Error $X$ estimates from the 20 sets of random samples for each window size, and shading indicates the $95 \%$ pointwise confidence intervals. The black boxes indicate the CLS range estimated from each spectra. CLSs indicated by hard corals (left column, foliose coral $[\mathrm{CF}]$ in orange, branching coral $[\mathrm{CB}]$ in blue) declined markedly from 2007 (top row) to 2010 (middle row). In the right column, spectra from soft coral (SC) is shown in orange, algae (A) in green, and dead coral (DC) (2007), and dead, algae-covered-coral (DCA) (2010 and 2011) are shown in dark blue.

The CLS estimated from sponges declined enough in 2011 to be detected as a regime shift; however the lack of a consistent pattern from the other morphotypes at this reef mean we would not be confident in identifying a regime shift here.
Hoga Buoy2 reef.-At Hoga Buoy 2 reef slope, there were a number of large changes in CLS derived from cover of hard corals over the period 2007 to 2011 (Fig. 10, Table 5). The CLS indicated by branching coral declined from $90 \mathrm{~cm}$ in 
TABLE 2. Blue Bowl reef characteristic length scale (CLS) estimates from one-dimensional sliding window Error $X$ spectra, and percent cover of each morphotype (in the 60-m transect).

\begin{tabular}{|c|c|c|c|c|c|c|}
\hline \multirow[b]{2}{*}{ Morphotype } & \multicolumn{3}{|c|}{ CLS $(\mathrm{cm})$} & \multicolumn{3}{|c|}{ Cover $(\%)$} \\
\hline & 2007 & 2010 & 2011 & 2007 & 2010 & 2011 \\
\hline Foliose coral & $55-70$ & $30-50(30)$ & $35-50(35)$ & 60.6 & 7.5 & 9.3 \\
\hline Branching coral & $60-70(65)$ & $30-40(30)$ & $40-55$ & 12.0 & 9.6 & 5.0 \\
\hline Dead coral (DC)/dead algae-covered coral (DCA) & $55-70(55)$ & $55-70(60)$ & $55-65(60)$ & $6.1(\mathrm{DC})$ & 58.3 (DCA) & $61.9(\mathrm{DCA})$ \\
\hline Soft coral & $45-65(45)$ & $35-45(35)$ & $30-40(30)$ & 7.9 & 5.8 & 2.9 \\
\hline Algae & $25-30$ & $35-45(45)$ & $40-55$ & 3.6 & 5.7 & 16.5 \\
\hline
\end{tabular}

Note: The CLS estimated from the Error $X$ spectra is stated as range of possible scales, but where possible, the most likely CLS is also given in parentheses.

2007, to about $50 \mathrm{~cm}$ in 2010. The next year (2011) it appeared to indicate multiple length scales: one at about $30 \mathrm{~cm}$ and possibly another at $60 \mathrm{~cm}$ or over. The CLS from cover of laminar coral declined by a similar magnitude: falling from around $65 \mathrm{~cm}$ in 2007 to around $15-20 \mathrm{~cm}$ in 2010 , and then remained low in 2011. Conversely, the CLS indicated by massive corals increased over the study period, from $40 \mathrm{~cm}$ in 2007 to $60 \mathrm{~cm}$ in 2011.

There appeared to be a slight increase in the CLS estimated from sponges from $20 \mathrm{~cm}$ to $40 \mathrm{~cm}$ between 2007 and 2011 at Hoga Buoy reef; however, the overlap in the estimated CLS ranges between years makes this uncertain. There was little apparent change in CLS determined from coralline algae, despite large changes in its cover. Similarly, there was no change in the CLS indicated by encrusting corals between 2007 and 2010, despite a large increase in the cover of this group, but there was a very small decline in the CLS estimated from it between 2010 and 2011. Finally, the CLS range estimates determined from soft coral were overlapping between years, making it difficult to confidently identify any change. Nevertheless, it appears likely that there was a decline in the CLS when estimated from soft coral between 2007 and 2011. Consistent changes in the CLS estimated from cover of hard corals between 2007 and 2010 mean that a regime shift was detected at this reef, despite the lack of clear change in CLS estimated from other morphotypes.

\section{DisCusSION}

We have successfully adapted the method for estimating the CLS of ecological systems (Keeling et al. 1997, Pascual and Levin 1999, Habeeb et al. 2005, Johnson 2009, Johnson et al. 2017) so that it can be used with one-dimensional spatial data (transects). Using this approach, we were able to estimate the 1D CLS for real and model systems based on the cover of most species or morphotypes that we tested. The results demonstrated that $1 \mathrm{D}$ CLSs are sensitive to the changes in network topology that we instigated. Using 1D CLS Error $X$ estimates, obtained with the sliding window method, we detected changes in ecological regimes in both the model and real world ecosystems examined. However there are caveats in the application of the method, which we detail in following sections.

The results of this study contribute important findings to ongoing discussion about what defines regime shifts and how to detect them (Mantua 2004, Rodionov 2005, Andersen et al. 2009, Doney and Sailley 2013). In our model test cases, it was circumstances where the structure of the interaction network changed (Test case 2 and Test case 4) that exhibited the clearest change in CLS. Conversely, in Test cases 1 and 3, where the network topology of the system was not changed, there was no clear change in CLS. The model test cases presented here provide a range of examples of ecosystem change, but it is not exhaustive. Further studies are necessary to determine whether these observations hold true in other test systems. Among the four coral reef sites, two had clear changes in the CLS, while the other two did not.

\section{Model test systems}

In the five-species model, the abundances of species changed in both test cases between the first and second regime, but only in the second test case did the changes manifest as a change in CLS. In Test case 1, although the reduction in growth rate of Species 5 did affect the abundances of other species, the form of the interaction network was still an intransitive loop, and there was no apparent change in CLS. However there was a change in magnitude (asymptote) of Error $X$ estimates obtained from Species 2 and 4. This difference in magnitude suggests a change in the system, but it is beyond the scope of this study to explore what kind of change it indicates.

In Test case 2, the interaction network began as an intransitive loop, but the interaction between Species 1 and 2 changed so that each could overgrow the other, changing the network topology. A feedback developed such that, as Species 2 had more opportunities for overgrowth, its numbers increased, which gave more overgrowth opportunities to Species 1 . Species 3 became scarce as Species 2 had more opportunities to overgrow it. This in turn meant Species 4 did well, and overgrew more of Species 5. Consequently, the cover of Species 1, 2 , and 4 increased while that of Species 3 and 5 decreased, and patch sizes increased overall. Thus, the change in the structure of the interaction network had significant implications for the scale of spatial self-organization in the system, which changed the CLS and, by definition, the shape of the attractor describing the dynamic (consistent with previous work; Habeeb et al. 2005, Johnson 2009, Johnson et al. 2017).

In the 20-species model, both test cases had the same randomly determined interaction network and had open recruitment to empty cells that were created by a low background level of disturbance. In Test case 3, the cessation of open recruitment did not change the form of the competitive interactions, but did result in the loss of the less competitive 

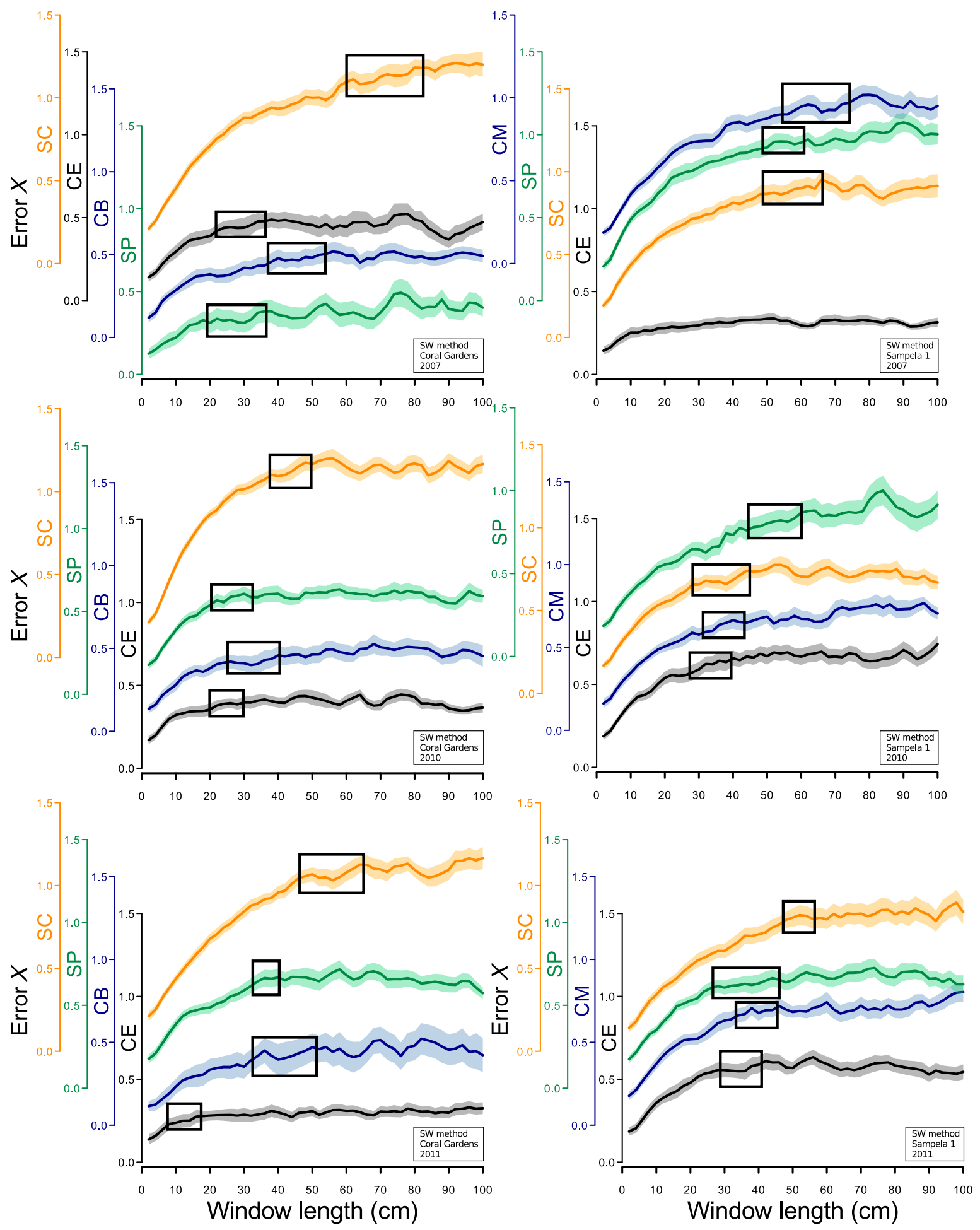

FIG. 9. Error $X$ spectra determined from cover of four morphotypes for each of Coral Gardens (left) and Sampela (right) reef slopes in 2007 (top), 2010 (middle), and 2011 (bottom). Soft coral (SC) spectra are shown in orange, sponges (SP) in green, encrusting coral (CE) in black. Blue lines are branching coral (CB) at Coral Gardens and massive coral (CM) at Sampela. The black boxes indicate the estimated CLS range from each spectra.

species from the landscape. The numbers of these species were originally low and their presence in the landscape was maintained solely by open recruitment to empty spaces. Their loss did not result in an observable change in the spatial scale of the system dynamics, implying that these species had little impact on the system dynamics overall. This suggests that the dynamics of this system were dominated by direct (overgrowth) interactions of key species rather 
TABLE 3. Coral Gardens reef CLS estimates from one-dimensional sliding window Error $X$ spectra, and percent cover for each morphotypes (in the 60-m transect).

\begin{tabular}{lcccrrr}
\hline \hline & \multicolumn{3}{c}{ CLS $(\mathrm{cm})$} & & \multicolumn{2}{c}{ Cover $(\%)$} \\
\cline { 2 - 4 } Morphotype & \multicolumn{1}{c}{2007} & 2010 & 2011 & 2007 & 2010 \\
\hline Encrusting coral & $20-35(30)$ & $20-35(25)$ & $10-20(15)$ & 3.0 & 3.6 \\
Soft coral & $60-80(60)$ & $40-55(45)$ & $45-65(65)$ & 35 & 20.6 & 31.8 \\
Sponge & $20-40(30)$ & $20-35(30)$ & $30-50$, or 70+(35) & 2.7 & 6.6 \\
Branching coral & $35-50(40)$ & $25-45(40)$ & 2.6 & 6.1 \\
\hline
\end{tabular}

Note: The CLS estimated from the Error $X$ spectra is stated as range of possible scales, but where possible, the most likely CLS is also given in parentheses.

TABLE 4. Sampela reef CLS estimates from one-dimensional sliding window Error $X$ spectra, and percent cover for each morphotypes (in the $60-\mathrm{m}$ transect).

\begin{tabular}{|c|c|c|c|c|c|c|}
\hline \multirow[b]{2}{*}{ Morphotype } & \multicolumn{3}{|c|}{ CLS $(\mathrm{cm})$} & \multicolumn{3}{|c|}{ Cover $(\%)$} \\
\hline & 2007 & 2010 & 2011 & 2007 & 2010 & 2011 \\
\hline Encrusting coral & $30-50(40)$ & $30-50(35)$ & $30-40(40)$ & 2.3 & 6.3 & 5.4 \\
\hline Soft coral & $50-65(50)$ & $30-45(40)$ & $45-55(50)$ & 11.2 & 7.6 & 6.6 \\
\hline Sponge & $50-60(50)$ & $50-60(50)$ & $25-45(25)$ & 14.3 & 8.1 & 6.3 \\
\hline Massive coral & $50-70(60)$ & $30-50(35)$ & $30-40(35)$ & 12.0 & 6.4 & 6.5 \\
\hline
\end{tabular}

Note: The CLS estimated from the Error $X$ spectra is stated as range of possible scales, but where possible, the most likely CLS is also given in parentheses.

than by the low levels of 'background' recruitment. The lack of change in CLS in this test case means we did not detect the changes as a regime shift. We interpret this to indicate that there was no regime shift in the system (that the loss of background recruitment of rare species did not substantially alter the system dynamic), rather than as a regime shift to a new attractor where the CLS is the same as in the original regime, although this is theoretically possible.

Test case 4 involved the arrival of a new species that was highly competitive with three species in the system, but did not interact directly with the other 17 species. In this case, one of the randomly selected species with which it interacted (Species 10) was highly competitive and among the most abundant species on the landscape. Consequently, the invader (Species 21) was able to dominate the landscape to such a point that its opportunities for overgrowing more cells became limited - it had reduced the abundance of those species it could overgrow, effectively blocking its own spread. Those species that did not directly interact with Species 21 remained present in the landscape, but their opportunities for interactions were likewise limited by the spatial dominance of Species 21, which they could not overgrow. These changes in the system reduced the CLS (which we estimated from Species 3 and 19), indicating that a regime shift occurred in this dynamic.

Using 2D short time-series prediction $r^{2}$ spectra, Johnson (2009) found that in each of these test cases a change in dynamics resulted in a change in the CLS of the system. The CLS values presented here are not directly comparable to those of Johnson because the analytical methods used are different (2D vs. 1D, short time series vs. sliding window, Prediction $r^{2}$ vs. Error $X$ ). Moreover, Johnson's 20-species models are not directly comparable to ours because their random interaction matrices are different. Nevertheless, for the test cases that are comparable (the five-species model test cases), and where we did detect a change in CLS using the 1D sliding window method (Test Case 2), the direction of the changes was consistent with those of Johnson (2009). It should be noted however, that while the network topology (i.e., nature of competitive interactions) of Johnson's fivespecies models and ours are the same, the CLSs indicated by the $1 \mathrm{D}$ analyses were generally smaller than those indicated by the $2 \mathrm{D}$ analyses. Also, the $1 \mathrm{D}$ analysis did not indicate a change in the CLS in both of the test cases with five species, where this was indicated by the 2D analysis. These differences in results between the 1D and 2D approaches using the same model system may reflect differences in the magnitude of variability captured by $2 \mathrm{D}$ and $1 \mathrm{D}$ windows. There is more spatial information in a $2 \mathrm{D}$ window than in a $1 \mathrm{D}$ window of the same length, and less variability between 2D windows than between $1 \mathrm{D}$ windows of the same length on the same landscape, and so the 2D CLS approach may be more sensitive to change. It is beyond the scope of this study to do a more in-depth comparison of $1 \mathrm{D}$ and 2D CLS. Future studies should aim to estimate and directly compare 2D and 1D CLS for the same system to better understand their sensitivities to changes in system topologies and dynamics.

The model test cases presented here provide examples of the kinds of changes to ecosystems that could plausibly induce a regime shift, however there are other mechanisms that can induce regime shift. Further work is required to determine whether the patterns we observed here are general to other systems. Our results do raise questions as to what constitutes a regime shift in this type of model system. The changes imposed in Test Cases 1 and 3 did not trigger changes in CLS in our model systems, but the same kind of change could have greater consequences in other systems. In our Test Case 1, the change in growth rate of a single species was not enough to change the CLS, while in other model 

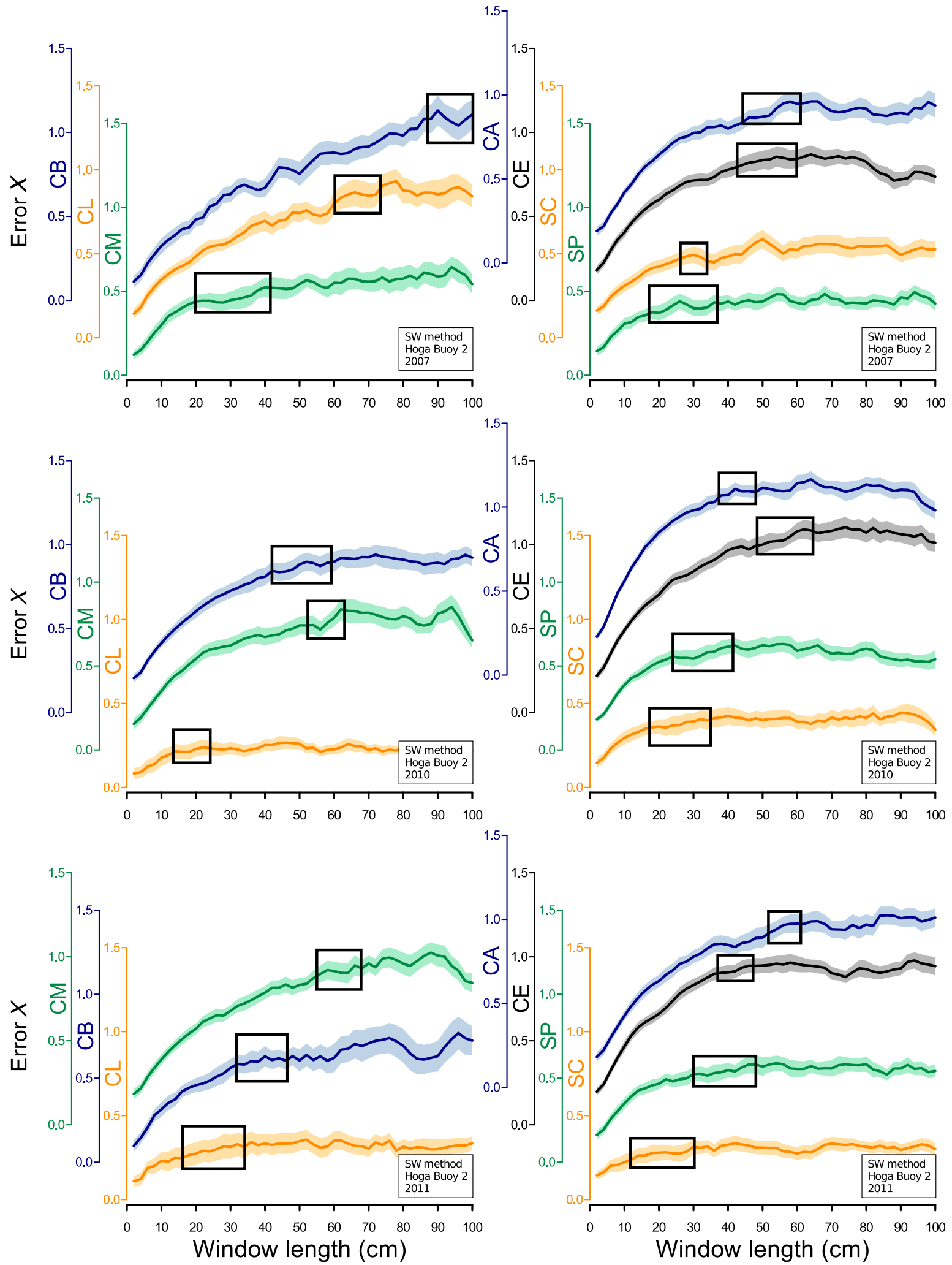

FIG. 10. Error $X$ curves determined from cover of seven morphotypes Hoga Buoy 2 reef slope for 2007 (top row), 2010 (middle), and 2011 (bottom row). Spectra from cover of hard coral morphotypes are presented in the left column: massive (CM, green), branching (CB, blue), and laminar (CL, orange), and other morphotypes (coralline algae [CA, blue], encrusting coral [CE, black], sponges [SP, green], and soft corals [SC, orange]) are presented on the right. The black boxes indicate the CLS range estimated from cover of each morphotype. 
TABLE 5. Hoga Buoy2 reef CLS estimates from one-dimensional sliding window Error $X$ spectra, and percent cover for each morphotypes (in the $60-\mathrm{m}$ transect).

\begin{tabular}{|c|c|c|c|c|c|c|}
\hline \multirow[b]{2}{*}{ Morphotype } & \multicolumn{3}{|c|}{ CLS $(\mathrm{cm})$} & \multicolumn{3}{|c|}{ Cover $(\%)$} \\
\hline & 2007 & 2010 & 2011 & 2007 & 2010 & 2011 \\
\hline Branching coral & $90+$ & $40-50(50)$ & $30-60(30$ or $60+)$ & 11.3 & 11.1 & 4.4 \\
\hline Laminar coral & $60-70(65)$ & $15-20$ & $15-30(15)$ & 6.9 & 0.95 & 1.2 \\
\hline Massive coral & $20-40(40)$ & 60 & $50-60(60)$ & 3.5 & 7.1 & 10.5 \\
\hline Coralline algae & $45-55(50)$ & $40-55(45)$ & $50-60(55)$ & 12.0 & 28.7 & 13.4 \\
\hline Encrusting coral & $40-55(50)$ & $45-60(60)$ & $40-50(45)$ & 10.5 & 17.6 & 15.0 \\
\hline Soft coral & $20-30(30)$ & $15-35(30)$ & $15-30(15)$ & 3.7 & 2.3 & 1.5 \\
\hline Sponge & $20-40(20)$ & $25-40(40)$ & $30-50(40)$ & 3.0 & 6.1 & 5.1 \\
\hline
\end{tabular}

Note: The CLS estimated from the Error $X$ spectra is stated as range of possible scales, but where possible, the most likely CLS is also given in parentheses.

systems changes in growth rate of a single species may have a substantial effect on the dynamics and CLS of the system (Johnson 2009), particularly if the species plays a key ecosystem role (e.g., keystone species, ecosystem engineer). Conversely, the arrival of a new 'invasive' species triggered a dramatic change in our model simulation (Test Case 4) since it happened to be highly competitive against very abundant species, but if a less competitive species was to arrive, or in a different system, there might be little observable impact on the system dynamics or on the CLS (Ricciardi et al. 2013).

\section{Coral reefs}

Coral reefs are open systems and are subject to a variety of ecological and physical processes (and stressors) operating at a wide range of spatial and temporal scales, and different reef organisms respond to the various processes (and stressors) to different degrees (Hughes et al. 2017). It is likely for this reason that CLSs estimated from different morphotypes at any one reef varied more than occurred in the model systems. For example, CLSs estimated from branching and laminar corals were often similar, indicating that they interact within the same dynamical subsystem of the coral reef community. The CLS estimates from coralline algae and encrusting coral were also often similar to each other, but tended to be different to the CLSs estimated from hard coral morphotypes. This indicates that different processes may determine the dynamics of these two groups, and that they interact within different subsystems, at least on the reefs examined as part of this study. Notably, for other benthic marine systems with closely interacting species, the CLSs estimated from a wide diversity of taxa representing a wide variety of life history characteristics are remarkably consistent (Johnson et al. 2017).

Changes in CLSs were evident at two of the coral reef sites (Blue Bowl and Hoga Buoy2), indicating a change not only in community structure but also in the nature of the system dynamics. The change in CLS for Blue Bowl is consistent with changes in community composition and ecosystem function described by Haapkylä et al. (2015) for this site, however the detection of a possible regime shift at Hoga Buoy 2 constitutes a new observation. Blue Bowl reef was a pristine site in 2007 with high $(74.4 \%)$ coral cover and a unique coral assemblage dominated by foliose corals (Haapkylä et al. 2015). However, between 2007 and 2010, there was a series of coral disease outbreaks, and consequently, a decline in coral cover. Over this time, the CLS determined from cover of hard corals decreased from 50 to $60 \mathrm{~cm}$, to about $30 \mathrm{~cm}$, making it similar to the CLS determined from cover of algae. This suggests a change in the system resulted in stronger interactions between hard corals and algae. At this site, filamentous algae overgrew many of the coral colonies that died completely or manifested patches of mortality (Haapkylä et al. 2015). Algal dominated reefs are widely thought to be an alternative stable state to coral dominated reefs (Done 1992, Mumby et al. 2007, Fong and Paul 2010, but see Mumby 2009, Fung et al. 2011), and given that we have observed such a clear change in CLSs at this site, it appears that this reef has transitioned to a new, algal-dominated attractor. Thus, while Haapkylä et al. (2015) were not able to ascertain whether the change they observed in assemblage structure constituted a regime shift, our results suggest clearly that it does.

At Hoga Buoy2 reef there was also a decline in the CLS coincident with a decline in cover of branching and laminar coral over the period 2007 to 2011. For branching coral in particular, the decline in CLS preceded the decline in cover, suggesting that there was a change in spatial structure and dynamics prior to the decline in cover. These declines were matched by an increase in the abundance of massive coral, and also in the CLS estimated from massive coral cover. Conversely, encrusting coral and coralline algae experienced large fluctuations in abundance but there was little change in CLS estimated from their cover, or from that of sponges. The changes in CLS derived from different groups of organism on this reef, together with the changes in composition, suggest that the dynamics changed in favor of benthic morphotypes that are more resistant to physical damage, and so may have flow on effects for the types of organisms inhabiting the reef. A regime shift was only indicated by the hard corals at this reef, and the CLSs estimated from their cover shifted to be closer to the CLSs estimated from the other morphotypes, indicating that perhaps these groups interacted more strongly after the regime shift.

Sampela1 and Coral Gardens reef slopes exhibited little change in CLS. At Sampela reef, there was possibly a decline in the CLS estimated from massive corals and sponges, however the uncertainty around those estimates makes it difficult to interpret. Of all of the reefs, Sampela is the closest to human settlement and was the most subject to human 
pressures, in particular high sedimentation and nutrient input (Haapkylä et al. 2009, 2015, Salinas-de-Leon et al. 2013). However, these pressures were present throughout the period of this study and prior to it, and so the reef had most likely already responded to these pressures, i.e., if there was a regime shift at this site, it occurred prior to 2007. Although all reefs in the area are subject to fishing pressures, Coral Gardens is considered a relatively pristine reef and the lack of clear change in the CLS suggests no significant shift in the dynamics of this reef over the period of our observation.

\section{Application of the 1D-CLS method}

A number of considerations regarding the use and application of CLSs from 1D data arise from this study, in particular for the length and resolution of the transect data used, the species investigated, and the interpretation of change. The length and spatial resolution of a transect dictates the range of spatial scales that can be considered in CLS estimation. Characteristic length scale estimation depends on having sufficient information about the spatial patterns of ecosystem components, whether they be species, morphotypes, or even habitats (see Habeeb et al. 2007), for each window size. The higher the spatial resolution, the more information will be available about spatial patterns at smaller scales and the more the landscape or transect can be subdivided into windows. Greater resolution therefore allows for smaller scales to be considered, which can facilitate identification of the CLS. Additionally, the sliding window method in particular is limited by the need for suitable replication of the larger window sizes. Longer transects enable replication of longer 1D windows along the transect (and greater replication of all window sizes). Therefore longer transects increase the maximum scale that can be detected. Shorter and/or lower resolution transects reduce the range of scales that can be considered, and will increase the risk that the actual CLS is outside that range (and thus remain undetected).

Initial testing showed that estimation of CLSs using the 1D sliding window method for Error $X$ spectra was not sensitive to choice of embedding dimension, spatial lag, or the number of nearest neighbours used in the prediction. However, we found some sensitivity to the maximum window size considered (discussed in more detail in Appendix S6). Another issue evident from this study is that rarer species may be less suitable to estimate CLSs using the 1D approach. Using the 1D methods, rare species have a high chance of being missed altogether by a transect, and so are more likely to be represented by zeros. Thus, for CLS estimation from 1D data, sufficient information about the patterns of rare species is unlikely to be captured and we recommend that more abundant species be investigated or that species are aggregated into functional groups. It appears that for real systems, it is important to estimate the system CLS using a range of species and then compare the CLSs to determine (1) which species are likely to be part of the same dynamic community and (2) whether changes implied by observations of one species are common to the whole community.

A potential limitation in using CLSs to detect regime shifts is that a shift is only detected if there is a clear change in the CLS that emerges from the system dynamics. It is theoretically possible that a shift could occur to a different regime that has the same emergent spatial scale. Further work is required to elucidate how likely this is to occur. The detectability of a shift might also be hampered in some cases given a level of subjectivity in estimating the CLS from the spectra. Because the exact point at which a variance spectrum plateaus can be difficult to pick, it is usual to identify the CLS as a range of possible window sizes. In cases where there appears to be a change in the CLS but the ranges overlap, there can be ambiguity in interpreting the difference as a regime shift. Given these limitations, this approach is conservative in detecting regime shifts in that it is only when there is a clear change in the CLS that we can assert strong evidence of a change in the regime. Since a change in state alone is insufficient evidence of regime shift (Johnson 2009), we are unaware of any other option to unambiguously interpret whether regime shifts have occurred. This method offers the potential for greater insight into the nature of ecosystem change, where a clear shift in the CLS is a powerful indicator that a regime shift has occurred. We recommend this approach be used as part of a toolbox of techniques for studying ecosystem change.

\section{Conclusion}

In conclusion, we have successfully adapted the 2D CLS estimation method for $1 \mathrm{D}$ data, and shown that the 1D method can be applied successfully to data from both model and real-world ecological systems. It is clear that the CLS can now be estimated for systems that are monitored using transects. Our results suggest that the characteristic length scale emerges as a useful metric for detecting regime shifts because it (1) has a value that depends on the dynamics of an entire community of interacting species, (2) can be estimated from a single, or very few, time steps, and (3) a change in the value of the CLS between time steps indicates a fundamental change in the ecosystem dynamics. Further work is required to test the generality of our observations in a broader range of model and real ecosystems.

\section{ACKNOWLEDGMENTS}

D. Ward is supported by an Australian Government Research Training Program Scholarship and a Quantitative Antarctic Science program top-up scholarship. This project was supported by the Australian Government's Cooperative Research Centres Programme through the Antarctic Climate and Ecosystem Cooperative Research Centre, by the Australian Antarctic Science Program (projects 4343 and 4347) and by NeCTAR. NeCTAR is supported by the Australian Government through the National Collaborative Research Infrastructure Strategy. Thanks to Mike Sumner for remote computing assistance, to Andrew Constable, and particularly Lewi Stone, whose helpful comments greatly improved the manuscript.

\section{Literature Cited}

Abarbanel, H. D. I. 1996. Analysis of observed chaotic data. Springer-Verlag, New York, New York, USA.

Ainsworth, C. H., and P. J. Mumby. 2015. Coral-algal phase shifts alter fish communities and reduce fisheries production. Global Change Biology 21:165-172.

Andersen, T., J. Carstensen, E. Hernandez-Garcia, and C. M. Duarte. 2009. Ecological thresholds and regime shifts: approaches to identification. Trends in Ecology and Evolution 24:49-57. 
Arya, S., D. Mount, S. E. Kemp, and G. Jefferis. 2017. RANN: Fast nearest neighbour search (wraps ANN library) Using L2 metric, R package version 2.5.1. https://CRAN.R-project.org/package=RANN

Beaugrand, G., P. C. Reid, F. Ibanez, J. A. Lindley, and M. Edwards. 2002. Reorganization of North Atlantic marine copepod biodiversity and climate. Science 296:1692-1694.

Beaugrand, G., et al. 2015. Synchronous marine pelagic regime shifts in the Northern Hemisphere. Philosophical Transactions of the Royal Society B 370:20130272.

Biggs, R., S. R. Carpenter, and W. A. Brock. 2009. Turning back from the brink: Detecting an impending regime shift in time to avert it. Proceedings of the National Academy of Sciences USA 106:826-831.

Biggs, R., T. Blenckner, C. Folke, L. Gordon, A. Norström, M. Nyrström, and G. Peterson. 2012. Regime shifts. Pages 609-617 in A. Hastings and L. J. Gross, editors. Encyclopedia of theoretical ecology. University of California Press, Berkeley, California, USA.

Dakos, V., et al. 2012. Methods for detecting early warnings of critical transitions in time series illustrated using simulated ecological data. PLoS ONE 7:e41010.

Di Lorenzo, E., and M. D. Ohman. 2013. A double-integration hypothesis to explain ocean ecosystem response to climate forcing. Proceedings of the National Academy of Sciences USA 110:2496-2499.

Done, T. J. 1992. Phase shifts in coral reef communities and their ecological significance. Hydrobiologia 247:121-132.

Doney, S. C., and S. F. Sailley. 2013. When an ecological regime shift is really just stochastic noise. Proceedings of the National Academy of Sciences USA 110:2438-2439.

Eddelbuettel, D., and W. Wu. 2016. RcppCNPy: Read-write support for NumPy files in R. Journal of Open Source Software 1:55.

Fong, P., and V. J. Paul. 2010. Coral reef algae. Pages 241-272 in Z. Dubinsky and N. Stambler, editors. Coral reefs: An ecosystem in transition. Springer, New York, New York, USA.

Fung, T., R. M. Seymour, and C. R. Johnson. 2011. Alternative stable states and phase shifts in coral reefs under anthropogenic stress. Ecology 92:967-982.

Graham, N. A. J., D. R. Bellwood, J. E. Cinner, T. P. Hughes, A. V. Norström, and M. Nyström. 2013. Managing resilience to reverse phase shifts in coral reefs. Frontiers in Ecology and the Environment 11:541-548.

Haapkylä, J., J. Melbourne-Thomas, and M. Flavell. 2015. The association between coral communities and disease assemblages in the Wakatobi Marine National Park, south-eastern Sulawesi, Indonesia. Marine and Freshwater Research 60:948-955.

Haapkylä, J., J. Melbourne-Thomas, M. Flavell, and D. Ward. 2016. Hoga coral reef benthic community transect data [Data set]. Australian Ocean Data Network. https://doi.org/10.4226/77/587e 953ebfdb3

Haapkylä, J., A. S. Seymour, J. Trebilco, and D. Smith. 2007. Coral disease prevalence and coral health in the Wakatobi Marine Park, south-east Sulawesi, Indonesia. Journal of the Marine Biological Association of the United Kingdom 87:403-414.

Haapkylä, J., R. K. F. Unsworth, A. S. Seymour, J. Melbourne-Thomas, M. Flavell, B. L. Willis, and D. J. Smith. 2009. Spatio-temporal coral disease dynamics in the Wakatobi Marine National Park, South-East Sulawesi, Indonesia. Diseases of Aquatic Organisms 87:105-115.

Habeeb, R. L., C. R. Johnson, S. Wotherspoon, and P. J. Mumby. 2007. Optimal scales to observe habitat dynamics: A coral reef example. Ecological Applications 17:641-647.

Habeeb, R. L., J. Trebilco, S. Wotherspoon, and C. R. Johnson. 2005. Determining natural scales of ecological systems. Ecological Monographs 75:467-487.

Hammond, M. P., and J. Kolasa. 2014. Spatial variation as a tool for inferring temporal variation and diagnosing types of mechanisms in ecosystems. PLoS ONE 9:e89245.

Hughes, T. P., et al. 2017. Coral reefs in the Anthropocene. Nature 546:82-90.

Johnson, C. 2002. Compete` An individual-based spatial modelling software to simulate ecological interactions and evolution in sessile organisms [Computer Software]. University of Tasmania (UTAS), School of Zoology. https://doi.org/10.4226/77/5b21a2dd2ba94

Johnson, C. R. 2009. Natural length scales of ecological systems: Applications at community and ecosystem levels. Ecology and Society $14: 7$

Johnson, C. R., R. H. Chabot, M. P. Marzloff, and S. Wotherspoon. 2017. Knowing when (not) to attempt ecological restoration. Restoration Ecology 25:140-147.

Kantz, H., and T. Schreiber. 2003. Nonlinear time series analysis. Second edition. Cambridge University Press, Cambridge, UK.

Keeling, M. J., I. Mezic, R. J. Hendry, J. McGlade, and D. A. Rand. 1997. Characteristic length scales of spatial models in ecology via fluctuation analysis. Philosophical Transactions of the Royal Society B 352:1589-1601.

Kéfi, S., V. Guttal, W. A. Brock, S. R. Carpenter, A. M. Ellison, V. N. Livina, D. A. Seekell, M. Scheffer, E. H. van Nes, and V. Dakos. 2014. Early warning signals of ecological transitions: methods for spatial patterns. PLoS ONE 9:e92097.

Litzow, M. A., A. J. Hobday, S. D. Frusher, P. Dann, and G. N. Tuck. 2016. Detecting regime shifts in marine systems with limited biological data: An example from southeast Australia. Progress in Oceanography 141:96-108.

Mantua, N. 2004. Methods for detecting regime shifts in large marine ecosystems: a review with approaches applied to North Pacific data. Progress in Oceanography 60:165-182.

Marcos-Nikolaus, P., J. M. Martin-Gonzalez, and R. V. Sole. 2002. Spatial forecasting: Detecting determinism from single snapshots. International Journal of Bifurcation and Chaos 12:369-376.

Möllmann, C., and R. Diekmann. 2012. Marine ecosystem regime shifts induced by climate and overfishing. Advances in Ecological Research 47:303-347.

Mumby, P. J. 2009. Phase shifts and the stability of macroalgal communities on Caribbean coral reefs. Coral Reefs 28:761-773.

Mumby, P. J., A. Hastings, and H. J. Edwards. 2007. Thresholds and the resilience of Caribbean coral reefs. Nature 450:98-101.

Nystrom, M., A. V. Norstrom, T. Blenckner, M. de la Torre-Castro, J. S. Eklof, C. Folke, H. Osterblom, R. S. Steneck, M. Thyresson, and M. Troell. 2012. Confronting feedbacks of degraded marine ecosystems. Ecosystems 15:695-710.

Pascual, M., and F. Guichard. 2005. Criticality and disturbance in spatial ecological systems. Trends in Ecology and Evolution 20:88-95.

Pascual, M., and S. A. Levin. 1999. From individuals to population densities: Searching for the intermediate scale of nontrivial determinism. Ecology 80:2225-2236.

Pecl, G. T., et al. 2017. Biodiversity redistribution under climate change: Impacts on ecosystems and human well-being. Science 355:eaai9214.

R Core Team. 2017. R: A language and environment for statistical computing. R Foundation for Statistical Computing, Vienna, Austria. www.r-project.org

Rand, D. A., and H. B. Wilson. 1995. Using spatio-temporal chaos and intermediate-scale determinism to quantify spatially extended ecosystems. Proceedings of the Royal Society B 259:111-117.

Reid, P. C., et al. 2015. Global impacts of the 1980s regime shift. Global Change Biology 22:682-703.

Ricciardi, A., M. F. Hoopes, M. P. Marchetti, and J. L. Lockwood. 2013. Progress toward understanding the ecological impacts of nonnative species. Ecological Monographs 83:263-282.

Rodionov, S. N. 2004. A sequential algorithm for testing climate regime shifts. Geophysical Research Letters 31:L09204.

Rodionov, S. 2005. A brief overview of the regime shift detection methods. Pages 17-24 in V. Velikova and N. Chipev, editors. Large-scale disturbances (regime shifts) and recovery in aquatic ecosystems: challenges for management toward sustainability. Proceedings of 2005 UNESCO-ROSTE/BAS Workshop on Regime Shifts, Varna, Bulgaria.

Rodionov, S., and J. E. Overland. 2005. Application of a sequential regime shift detection method to the Bering Sea ecosystem. ICES Journal of Marine Science 62:328-332. 
RStudio Team. 2015. RStudio: Integrated development for R. RStudio Inc, Boston, Massachusetts, USA.

Salinas-de-Leon, P., J. J. Bell, C. Dryden, and D. J. Smith. 2013. Temporal and spatial variability in coral recruitment on two Indonesian coral reefs: consistently lower recruitment to a degraded reef. Marine Biology 160:97-105.

Scheffer, M., and S. R. Carpenter. 2003. Catastrophic regime shifts in ecosystems: linking theory to observation. Trends in Ecology and Evolution 18:648-656.

Scheffer, M., S. Carpenter, J. A. Foley, C. Folke, and B. Walker. 2001. Catastrophic shifts in ecosystems. Nature 413:591-596.

Scheffer, M., et al. 2012. Anticipating critical transitions. Science 338:344-348

Scheffer, M., S. R. Carpenter, V. Dakos, and E. van Nes. 2015. Generic indicators of ecological resilience: inferring the chance of a critical transition. Annual Review of Ecology, Evolution, and Systematics 46:145-167.

Selkoe, K. A., et al. 2015. Principles for managing marine ecosystems prone to tipping points. Ecosystem Health and Sustainability $1: \operatorname{art} 17$.

Sousa, W. P. 1984. The role of disturbance in natural communities. Annual Review of Ecology and Systematics 15:353-391.

Sugihara, G., and R. M. May. 1990. Nonlinear forecasting as a way of distinguishing chaos from measurement error in time-series. Nature 344:734-741.
Takens, F. 1981. Detecting strange attractors in turbulence. Pages 366-381 in D. A. Rand and L. S. Young, editors. Dynamical systems and turbulence, Warwick 1980, Lecture notes in mathematics. Springer-Verlag, Berlin, Germany.

Travis, J., F. Coleman, P. J. Auster, P. M. Cury, J. A. Estes, J. Orensanz, C. H. Peterson, M. E. Power, R. S. Steneck, and J. T. Wootton. 2014. Integrating the invisible fabric of nature into fisheries management. Proceedings of the National Academy of Sciences USA 111:581-584.

Trebilco, J. 2002. Estimating characteristic length scales of dynamic biological systems: removing the need for long time series. Honours Thesis. University of Tasmania, Hobart, Tasmania, Australia. http://eprints.utas.edu.au/23658/

Turner, M. G. 2010. Disturbance and landscape dynamics in a changing world. Ecology 91:2833-2849.

Walther, G. R. 2010. Community and ecosystem responses to recent climate change. Philosophical Transactions of the Royal Society B 365:2019-2024.

Walther, G.-R., E. Post, P. Convey, A. Menzel, C. Parmesank, T. J. C. Beebee, J.-M. Fromentin, O. Hoegh-Guldberg, and F. Bairlein. 2002. Ecological responses to recent climate change. Nature 416:389-395.

deYoung, B., M. Barange, G. Beaugrand, R. Harris, R. I. Perry, M. Scheffer, and F. Werner. 2008. Regime shifts in marine ecosystems: detection, prediction and management. Trends in Ecology and Evolution 23:402-409.

SUPPORTING INFORMATION

Additional supporting information may be found online at: http://onlinelibrary.wiley.com/doi/10.1002/ecm.1312/full

\section{Data Availability}

The coral reef data are available through the Australian Ocean Data Network: https://doi.org/10.4226/77/587e953ebfdb3. The code presented here are available as the R package "CLS1D”, accessible through Zenodo: https://doi.org/10.5281/zenodo.1218773. 